\title{
CONTROL OF AN IDEAL ACTIVATED SLUDGE PROCESS IN WASTEWATER TREATMENT VIA AN ODE-PDE MODEL
}

\author{
STEFAN DIEHL* AND SEBASTIAN FARÅS
}

\begin{abstract}
The activated sludge process (ASP), found in most wastewater treatment plants, consists basically of a biological reactor followed by a sedimentation tank, which has one inlet and two outlets. The purpose of the ASP is to reduce organic material and dissolved nutrients (substrate) in the incoming wastewater by means of activated sludge (microorganisms). The major part of the discharged flow through the bottom outlet of the sedimentation tank is recirculated to the reactor, so that the biomass is reused. Only two material components are considered; the soluble substrate and the particulate sludge. The biological reactions are modelled by two nonlinear ordinary differential equations and the continuous sedimentation process by two hyperbolic partial differential equations (PDEs), which have coefficients that are discontinuous functions in space due to the inlet and outlets. In contrast to previously published modelling-control aspects of the ASP, the theory for such PDEs is utilized. It is proved that the most desired steady-state solutions can be parameterized by a natural control variable; the ratio of the recirculating volumetric flow to the input flow. This knowledge is a key ingredient in a two-variable regulator, with which the effluent dissolved nutrients concentration and the concentration profile in the sedimentation tank are controlled. Theoretical results are supported by simulations.
\end{abstract}

\section{Introduction}

The need for efficient wastewater treatment plants in terms of low effluent concentrations of organic material and dissolved nutrients has been driving research in applied mathematics and control and water engineering. Of particular interest is the control of the activated sludge process (ASP), found in most wastewater treatment plants. It consists basically of a biological reactor and a sedimentation tank, or settler; see Figure 1. The purification is done in the biological reactor where microorganisms, the biomass, consumes and decomposes nutrients, the soluble material, under the influence of oxygen. The biomass forms flocculated particles, which are separated from the water continuously in the settler. Of the two compartments, the biological reactor has received most attention in the literature. We focus on capturing how the nonlinearities of the continuous-sedimentation process influence the ASP and start with a model as simple as possible; however, without imposing any heuristic assumptions on the solution of the model equations of the settler, which is often seen in previous publications.

Date: December 19, 2012.

Key words and phrases. partial differential equation; regulator; controller; clarifier-thickener; settler; continuous sedimentation.

${ }^{*}$ Corresponding author. Telephone +46 462220920 .

Centre for Mathematical Sciences, Lund University, P.O. Box 118, S-221 00 Lund, Sweden. E-mail: diehl@maths.lth.se, faras@maths.lth.se. 


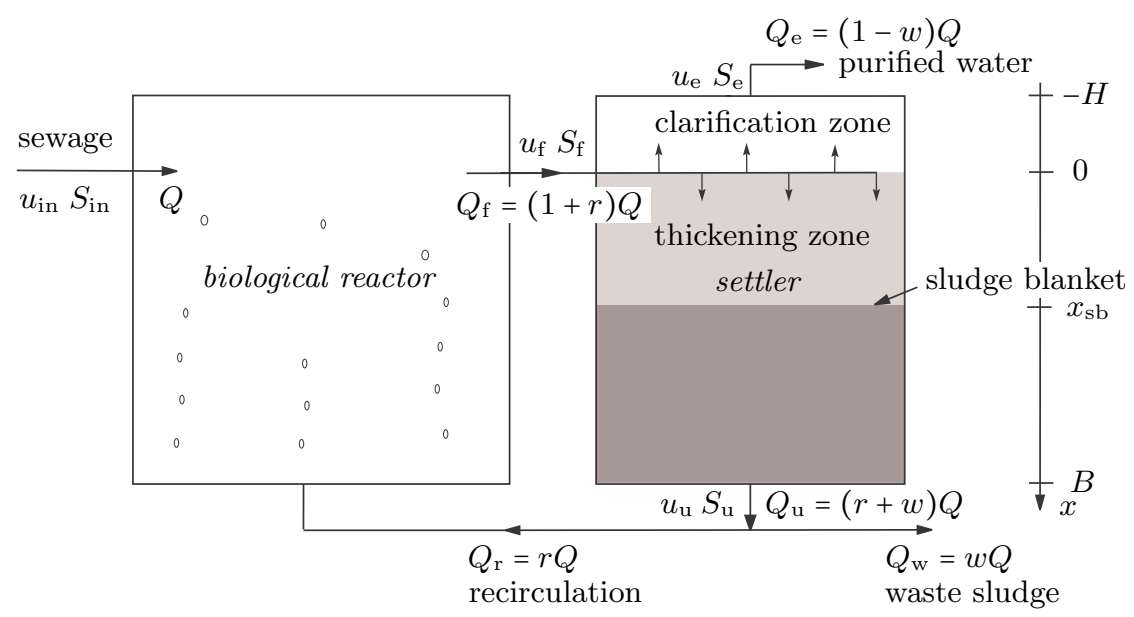

FiguRE 1. The ideal activated sludge process (ASP) consists of a completely mixed biological reactor and a one-dimensional sedimentation tank (settler). The settler consists of a clarification zone corresponding to the interval $(-H, 0)$ and a thickening zone in the interval $(0, B)$. All variables except $H$ and $B$ may depend on time. The indices stand for $\mathrm{f}=$ feed, $\mathrm{e}=$ effluent, $\mathrm{u}=$ underflow, $\mathrm{r}=$ recycle and $\mathrm{w}=$ waste.

A conventional model for the biological reactions consists of 13 ordinary differential equations (ODEs) modelling several soluble and particulate components [32]. Since the concentration of the particles in the settler depends on both time and space, the conservation of mass leads to a partial differential equation (PDE). For simplicity, one-dimensional settler models are usually considered and we assume that the hindered settling velocity of particles is a decreasing function of the concentration. Then the PDE is nonlinear with a spatially discontinuous flux function and a source term because of the inflow at $x=0$ and outflows at $x=-H$ and $x=B$; see Figure 1:

$$
\frac{\partial u}{\partial t}+\frac{\partial}{\partial x} F(u, x, r, w, Q)=\frac{Q_{\mathrm{f}} u_{\mathrm{f}}}{A} \delta(x),
$$

where the notation is properly defined in Section 2. For the analysis of this type of PDE regarding well-posedness and reliable numerical schemes; see [9, 22, 35] and references therein. Equation (1) is the most simple model of continuous sedimentation one can find in the literature, yielding reliable solutions that satisfy the conservation of mass also at inlet and outlets, without imposing any ad hoc assumptions on the solution; see [14]. There exists (several) publications with even simpler "models" of the settler, for example, "point settlers" with either a completely mixed tank or some assumed relation between the underflow and feed concentrations. We regard such approaches as too unrealistic since they do not model the gravity sedimentation of suspended particles in a liquid.

From a modelling point of view, the most simple model for an ideal ASP consists of only two material components; a soluble substrate and a particulate biomass component, and only one constitutive assumption for each compartment; the biomass 
growth in the biological reactor and the settling velocity for the settler. This results in a coupled system of two ODEs for the reactor and two PDEs for the settler. The only proper analysis of such a system of equations found in the literature is the preceding paper [23] by the authors. In particular, there exists no published ASP controller that includes the control of the continuous-sedimentation process. It is the main purpose of the present article to investigate the possibilities for the simultaneous control of the settler behaviour and the effluent soluble substrate concentration.

Modelling and control of the ASP have been challenging for several decades. An early approach taking into account the settler in a simulation model was done by Attir and Denn [4], who used the same reduced-order model as the present article. Otherwise, the complex nonlinear processes in the biological reactor have received most attention; see e.g. $[25,26,27,44,46]$. Some recent works focus on different control aspects of the ASP, e.g. [2, 6, 30, 34, 36, 38, 45, 50]. Their ODE models and control strategies are focused on the biological reactions and are more sophisticated than the simple model here. However, they all contain very simplified (too unrealistic) models for the sedimentation process. Because of these two facts, a comparison with the results here are not meaningful. Instead, the present work should be seen as a complement to those publications (see the concluding discussion in Section 7).

Several published results are based on simulations and case studies, e.g. [1, 11, 29, $31,33,47,49,51]$; however, simulation models cannot produce general connections between variables in a nonlinear ASP model (including the settler), which is the purpose to investigate in the present article. Many of these references also contain numerical schemes with heuristic ingredients for the numerical implementation of PDEs like (1) that make them unreliable [7]. Interesting modelling and control approaches have been presented by Lee et al. [39, 40, 41, 42]. Their model is more elaborate than others with linear PDEs describing the movement and turbulence in the biological reactor for each component. They present a general algorithm for robust model-order reduction in [40] and a robust multi-variable controller for the biological processes in [41]. Both approaches rely on linearizations at steady states. Hence, they neither capture the nonlinear behaviour of the sedimentation process, nor present any control of it, which is the purpose of the present article. The difficulties of controlling the ASP are discussed recently by Ekama [28], who stresses the importance of controlling the sludge age. The influence of the settler is then of great importance. Araujo et al. [3] describe a systematic sensitivity analysis of optimal economic operation of an ASP model in which steady-state equations for the PDE (1) are used.

Available reliable numerical schemes, e.g. [7, 9], are not sufficient for control of the continuous-sedimentation process. Some control strategies can be found in [5, $10,12,13,15,43,48]$. They all deal with the control of a concentration discontinuity in the thickening zone, called the sludge blanket (see Figure 1), under normal operating conditions, which means that the effluent concentration of biomass is zero and the underflow concentration is high. Exact steady-state solutions of (1) and transitions between these (step responses) can be constructed and thereby also control issues resolved [16, 17, 18, 19]. Based on these results, Diehl [20] developed a robust regulator for the model PDE (1) for an uncoupled settler. 
Despite the simple reduced-order ASP model used here, the analysis of the equations has turned out to be rather complex, mostly because of the so-called limiting flux in the thickening zone of the settler - a well-known concept in the so-called solids-flux theory and which is nicely captured in the theory for the model PDE (1); see [21]. One idea behind our development of a strategy for automatic control of the ASP is to find the (nonlinear) steady-state relationships between the different variables in the model. In [23], the authors have classified all steady states for given input variables and fixed control variables $(r$ and $w$ in Figure 1). It has been sorted out for what combination of input values a trivial steady state exists (wash-out solution, no biomass in the ASP) and when a "normal" solution exists. Furthermore, the stability of those solutions with respect to disturbances in the reactor was investigated. It turned out that the normal solution is globally stable. In each normal steady state, the settler may be critically, under- or overloaded depending on the values of the input and control variables. In the present article, we derive the conditions on the control variables $r$ and $w$ which make the normal steady state have a critically loaded settler, which is the desired state with the possibility of a sludge blanket in the thickening zone.

One result here is that the steady-state substrate concentration, which is the same in the entire ASP, can be described as a function of $r$ and $w$ under the constraint that the settler is critically loaded. When such a function is known, it can be used for manual control and investigation with respect to changes in the input variables. The role of the biomass sludge age for steady-state solutions and a demonstration of manual control have been presented in [24]; however, without the full proofs. Here we provide all proofs for the manual control.

The main contribution here is a two-variable regulator for the control of the substrate concentration $S_{\mathrm{f}}$ and the sludge blanket level. The reference value $S^{\text {ref }}$ is converted to reference values $r^{\text {ref }}$ and $w^{\text {ref }}$ for the regulator by utilizing the abovementioned function between the substrate concentration $S_{\mathrm{f}}$ and $r$ and $w$. Feedback control on the mass is used to keep the sludge blanket at a reference level.

The paper is organized as follows. The ASP model is described shortly in Section 2 together with a control objective. In Section 3, the algebraic equations for obtaining the "normal" steady-state solution are reviewed from [23]. Only some of such steady states have a critically loaded settler with a sludge blanket in the thickening zone. Section 4 contains the result that the subset of desired steady states is characterized by a curve in the plane of the two control variables $(r$ and $w)$, the control curve. The dependencies of the input variables on $r$ and $w$ along the control curve are given in Section 5 . Section 6 contains a strategy for automatic control by means of a two-variable regulator, some results on the closed-loop system, simulations and discussions on the tuning and disturbances. Proofs of most of the theorems are placed in the Appendix.

\section{THE REDUCED-ORDER MODEL FOR THE ASP}

The ASP model, shown in Figure 1, is described comprehensively in [23] and briefly here. There is one substrate component, the soluble material, represented by the $S$-variables, and one biomass component, the particulate material, represented by $u$. The conservation of mass implies the following model equations:

$$
V \frac{d S_{\mathrm{f}}}{d t}=Q S_{\mathrm{in}}+r Q S_{\mathrm{u}}-(1+r) Q S_{\mathrm{f}}-V \frac{\mu\left(S_{\mathrm{f}}\right)}{Y} u_{\mathrm{f}}
$$




$$
\begin{aligned}
& V \frac{d u_{\mathrm{f}}}{d t}=Q u_{\mathrm{in}}+r Q u_{\mathrm{u}}-(1+r) Q u_{\mathrm{f}}+V\left(\mu\left(S_{\mathrm{f}}\right)-b\right) u_{\mathrm{f}}, \\
& A \frac{\partial S}{\partial t}+A \frac{\partial}{\partial x}\left(F^{\mathrm{s}}(S, x, r, w, Q)\right)=(1+r) Q S_{\mathrm{f}} \delta(x), \\
& A \frac{\partial u}{\partial t}+A \frac{\partial}{\partial x}(F(u, x, r, w, Q))=(1+r) Q u_{\mathrm{f}} \delta(x) .
\end{aligned}
$$

Equations (2)-(3) model the processes in the biological reactor and (4)-(5) model the settler. The variables are given in Table 1 .

TABLE 1. The notation for the ASP model equations (2)-(5).

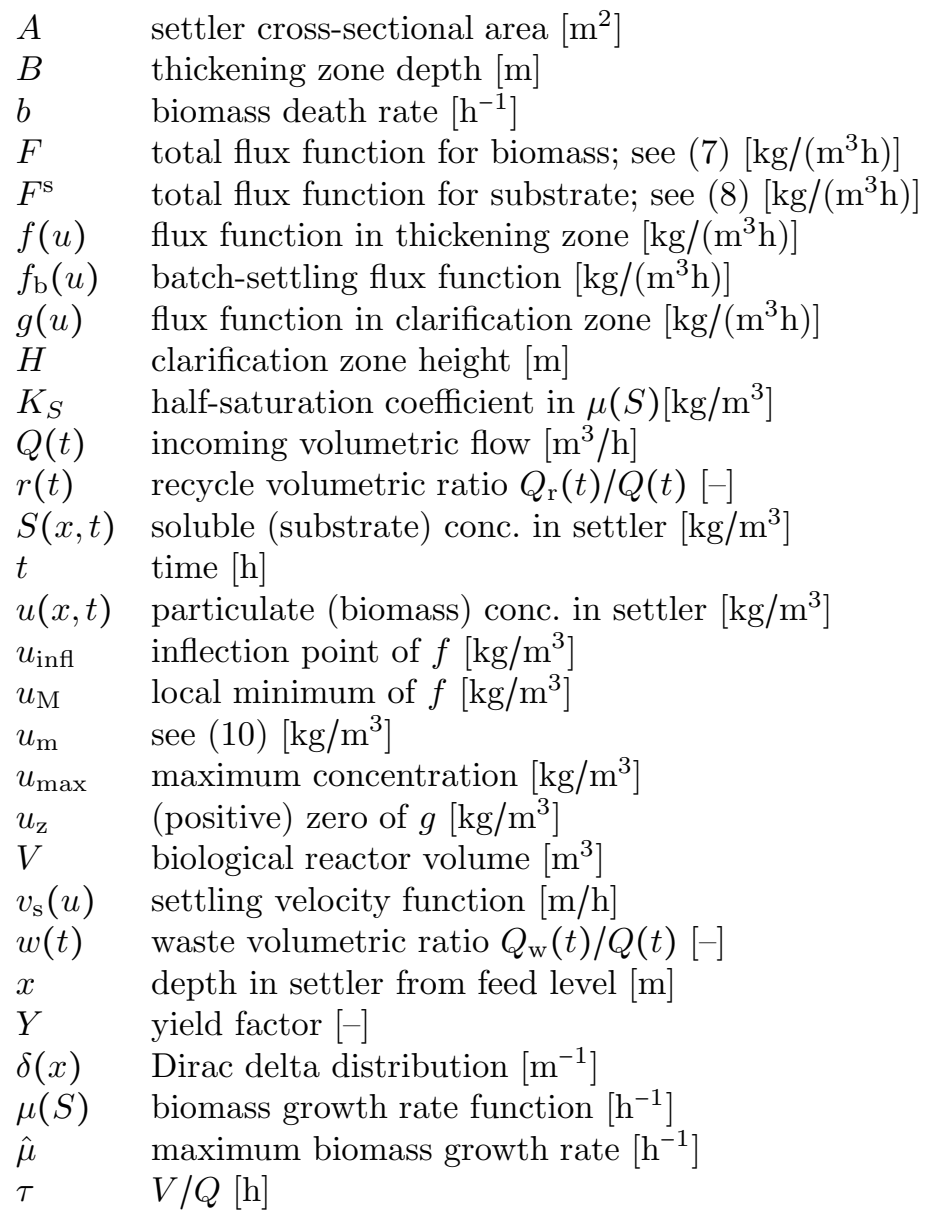

The constitutive assumption for the biological reactor is that the biomass growth, $\mu$, is a function of the substrate concentration only, since it is assumed that there is a sufficient supply of oxygen. We assume that $\mu \in C^{2}$ and that it satisfies the following properties, where $\hat{\mu}$ is a constant:

$$
\begin{aligned}
& \mu(0)=0, \quad \lim _{S \rightarrow \infty} \mu(S)=\hat{\mu}, \\
& 0<\mu^{\prime}(S)<\infty \quad \text { and } \quad \mu^{\prime \prime}(S)<0 \text { for } S \geq 0 .
\end{aligned}
$$


The constitutive assumption for the gravity settling is the one by Kynch [37]: the settling velocity $v_{\mathrm{s}}$ depends only on the local concentration $u$. We assume that $v_{\mathrm{s}} \in C^{2}$ is a decreasing function satisfying $v_{\mathrm{s}}\left(u_{\max }\right)=0$, where $u_{\max }$ is the maximum packing concentration. The batch-settling flux function $f_{\mathrm{b}}(u):=u v_{\mathrm{s}}(u) \in C^{2}$ is assumed to have precisely one inflection point $u_{\text {inf }} \in\left(0, u_{\max }\right)$.

The dimensionless recycle and waste volumetric ratios, $r:=Q_{\mathrm{r}} / Q$ and $w:=Q_{\mathrm{w}} / Q$, are the control variables and the flux functions of (4)-(5) are

$$
\begin{aligned}
F(u, x, r, w, Q): & = \begin{cases}-\frac{(1-w) Q}{A} u, & x<-H, \\
g(u,(1-w) Q), & -H<x<0, \\
f(u,(r+w) Q), & 0<x<B, \\
\frac{(r+w) Q}{A} u, & x>B,\end{cases} \\
F^{\mathrm{S}}(S, x, r, w, Q): & \begin{cases}-\frac{(1-w) Q}{A} S, & x<0, \\
\frac{(r+w) Q}{A} S, & x>0,\end{cases}
\end{aligned}
$$

where

$$
\begin{array}{ll}
g(u)=g\left(u, Q_{\mathrm{e}}\right):=f_{\mathrm{b}}(u)-\frac{Q_{\mathrm{e}}}{A} u & \text { flux in clarification zone, } \\
f(u)=f\left(u, Q_{\mathrm{u}}\right):=f_{\mathrm{b}}(u)+\frac{Q_{\mathrm{u}}}{A} u & \text { flux in thickening zone. }
\end{array}
$$

There are particular characteristic concentrations that appear in the solution. Let $u_{\mathrm{z}}>0$ be the unique positive zero of $g$. Define

$$
\bar{Q}_{\mathrm{u}}:=-A f_{\mathrm{b}}^{\prime}\left(u_{\max }\right), \quad \overline{\bar{Q}}_{\mathrm{u}}:=-A f_{\mathrm{b}}^{\prime}\left(u_{\mathrm{infl}}\right),
$$

which are the volumetric flows such that the slope of $f$ is zero at $u_{\text {max }}$ and $u_{\text {infl }}$, respectively. The local minimizer of $f$, denoted $u_{\mathrm{M}}$, on the right of $u_{\mathrm{inf}}$ plays an important role in the behaviour of the process. For intermediate values of $Q_{\mathrm{u}}$, i.e., $\bar{Q}_{\mathrm{u}}<Q_{\mathrm{u}}<\overline{\bar{Q}}_{\mathrm{u}}$, we have $0=f^{\prime}\left(u_{\mathrm{M}}\right)=f_{\mathrm{b}}^{\prime}\left(u_{\mathrm{M}}\right)+Q_{\mathrm{u}} / A$. To obtain a definition for all values of $Q_{\mathrm{u}}$ we define the restriction $\tilde{f}_{\mathrm{b}}=\left.f_{\mathrm{b}}\right|_{\left(u_{\text {infl }}, u_{\max }\right)}$. Then $\tilde{f}_{\mathrm{b}}^{\prime}$ is increasing and we define

$$
u_{\mathrm{M}}\left(Q_{\mathrm{u}}\right):= \begin{cases}u_{\max }, & 0 \leq Q_{\mathrm{u}} \leq \bar{Q}_{\mathrm{u}}, \\ \left(\tilde{f}_{\mathrm{b}}^{\prime}\right)^{-1}\left(-Q_{\mathrm{u}} / A\right), & \bar{Q}_{\mathrm{u}}<Q_{\mathrm{u}}<\overline{\bar{Q}}_{\mathrm{u}}, \\ u_{\mathrm{infl}}, & Q_{\mathrm{u}} \geq \overline{\bar{Q}}_{\mathrm{u}} .\end{cases}
$$

Given $u_{\mathrm{M}}$, define $u_{\mathrm{m}}$ as the unique concentration satisfying

$$
f\left(u_{\mathrm{m}}\right)=f\left(u_{\mathrm{M}}\right), \quad 0 \leq u_{\mathrm{m}} \leq u_{\text {infl. }} .
$$

All steady-state solutions of Eq. (5) have been classified in [16]. The most desired steady-state solution has zero concentration in the clarification zone and a sludge blanket in the thickening zone with the concentrations $u_{\mathrm{m}}$ above and $u_{\mathrm{M}}$ below. The settler is then said to work in optimal operation in steady state. The following definition also covers dynamic solutions.

Definition 2.1. The settler is said to be in optimal operation at time $t$ if $Q_{\mathrm{u}}(t)<$ $\overline{\bar{Q}}_{\mathrm{u}}$ and the solution of (5) satisfies:

- $u(x, t)=0$ for $-H<x<0$, 
- there exists a level, the sludge blanket level, $x_{\mathrm{sb}}(t) \in(0, B)$ such that

$$
u(x, t) \in \begin{cases}{\left[0, u_{\text {infl }}\right),} & 0<x<x_{\mathrm{sb}}(t) \\ {\left[u_{\text {infl }}, u_{\text {max }}\right],} & x_{\mathrm{sb}}(t)<x<B .\end{cases}
$$

Definition 2.2. The control objective for the ideal ASP is that the effluent substrate concentration $S_{\mathrm{e}}$ should lie close to a given reference value $S^{\mathrm{ref}}$ and the settler should be in optimal operation with the sludge blanket level $x_{\mathrm{sb}}$ close to a given reference value $x_{\mathrm{sb}}^{\mathrm{ref}}$.

If the control objective is satisfied, then the underflow concentration $u_{\mathrm{u}}$ is high, which is preferable in order to keep a low recirculation ratio $r$ and a concentrated waste sludge. To prevent overflow, the waste ratio $w$ has to be nonzero in steady state since the microorganisms grow in the biological reactor. On the other hand, $w$ should be small to minimize the amount of waste sludge for practical, economical and environmental reasons. Furthermore, a large value of $r$ may cause unwanted turbulence in the settler, and implies a high energy consumption to recirculate the suspension within the plant. Orders of magnitude are usually $r \approx 1$ and $w \approx 0.01$.

For graphs and simulations, we have used $v_{\mathrm{s}}(u)=10\left(e^{-0.35 u}-e^{-0.35 u_{\max }}\right) \mathrm{m} / \mathrm{h}$, the Monod growth rate function $\mu(S)=\hat{\mu} S /\left(S+K_{S}\right)$, and the following parameter values and constants, unless otherwise stated:

$$
\begin{array}{llll}
Q=1000 \mathrm{~m}^{3} / \mathrm{h}, & S_{\text {in }}=0.2 \mathrm{~kg} / \mathrm{m}^{3}, & u_{\mathrm{in}}=0.1 \mathrm{~kg} / \mathrm{m}^{3}, & V=2000 \mathrm{~m}^{3}, \\
A=1500 \mathrm{~m}^{2}, & Y=0.7, & b=0.01 \mathrm{~h}^{-1}, & \hat{\mu}=0.1 \mathrm{~h}^{-1}, \\
K_{S}=0.15 \mathrm{~kg} / \mathrm{m}^{3}, & r=1, & w=0.01, & u_{\max }=15 \mathrm{~kg} / \mathrm{m}^{3} .
\end{array}
$$

\section{The STEAdy States OF THE ASP MOdeL}

In [23], all steady-state solutions of the ASP are classified for constant inputs and control variables. Under normal operating conditions, i.e., for most realistic values of the parameters and constants in the constitutive assumptions, there exists a unique non-trivial and stable steady-state solution, which we call the normal solution. It has the same constant soluble concentration in the entire ASP, i.e. $S=S_{\mathrm{f}}=S_{\mathrm{e}}=S_{\mathrm{u}}$. To present the steady-state equations, we define the feed flux $\Phi_{\mathrm{f}}:=Q_{\mathrm{f}} u_{\mathrm{f}}$, the feed flux function $\widetilde{\Phi_{\mathrm{f}}}\left(u_{\mathrm{f}}, r, Q\right):=(1+r) Q u_{\mathrm{f}}$ and the limiting flux function [13]:

$$
\begin{aligned}
\Phi_{\lim }\left(u, Q_{\mathrm{u}}\right):=\min _{u \leq v \leq u_{\max }} A f\left(v, Q_{\mathrm{u}}\right) & \\
= & \begin{cases}A f\left(u, Q_{\mathrm{u}}\right), & u \in\left[0, u_{\mathrm{m}}\left(Q_{\mathrm{u}}\right)\right] \cup\left[u_{\mathrm{M}}\left(Q_{\mathrm{u}}\right), u_{\max }\right], \\
A f\left(u_{\mathrm{M}}\left(Q_{\mathrm{u}}\right), Q_{\mathrm{u}}\right), & u \in\left(u_{\mathrm{m}}\left(Q_{\mathrm{u}}\right), u_{\mathrm{M}}\left(Q_{\mathrm{u}}\right)\right) .\end{cases}
\end{aligned}
$$

For constant $r, w$ and $Q$, we write shorter $\widetilde{\Phi_{\mathrm{f}}}\left(u_{\mathrm{f}}\right)$, etc. Furthermore, we define

$$
\begin{aligned}
& U_{\mathrm{f}}(S):=\frac{Y}{\mu(S) \tau}\left(S_{\mathrm{in}}-S\right), \quad 0<S \leq S_{\mathrm{in}}, \\
& L(S): \widetilde{\Phi_{\mathrm{f}}}\left(U_{\mathrm{f}}(S)\right)\left(1+\frac{b \tau}{1+r}\right) \\
& \quad-\frac{r}{r+w} \min \left(\widetilde{\Phi_{\mathrm{f}}}\left(U_{\mathrm{f}}(S)\right), \Phi_{\lim }\left(U_{\mathrm{f}}(S),(r+w) Q\right)\right)+Y Q S, \quad 0<S \leq S_{\mathrm{in}}, \\
& \alpha(r, w):=\frac{w(1+r)}{r+w}, \quad r>0,0 \leq w<1 .
\end{aligned}
$$


Since the properties (6) imply

$$
U_{\mathrm{f}}^{\prime}(S)=-\frac{Y}{\mu(S)^{2} \tau}\left(\mu(S)+\left(S_{\mathrm{in}}-S\right) \mu^{\prime}(S)\right)<0, \quad 0<S \leq S_{\mathrm{in}},
$$

the inverse of $U_{\mathrm{f}}$ exists and we can define $S_{\min }:=U_{\mathrm{f}}^{-1}\left(u_{\max }\right)$, which is the lower bound for the substrate concentration $S$.

The conditions for a normal steady-state solution are given in [23, Theorem 3.2], from which we subtract the following.

Theorem 3.1. For every fixed $Q>0, S_{\text {in }}>0, u_{\text {in }} \geq 0, r>0$ and $0 \leq w<1$, which satisfy the inequalities

$$
\begin{aligned}
v_{\mathrm{s}}(0) & >\frac{Q_{\mathrm{e}}}{A}=\frac{(1-w) Q}{A}, \\
Y S_{\mathrm{in}}+u_{\mathrm{in}} & \leq Y S_{\min }+u_{\max }(1+b \tau),
\end{aligned}
$$

a normal solution exists if and only if either $u_{\mathrm{in}}>0$ holds, or $u_{\mathrm{in}}=0$ and

$$
\left(\mu\left(S_{\mathrm{in}}\right)-b\right) \tau>\alpha(r, w)
$$

hold. (Technical conditions for uniqueness can be found in [23, Theorem 3.2].) The normal solution satisfies $S \in\left[S_{\min }, S_{\mathrm{in}}\right), u_{\mathrm{f}}, u_{\mathrm{u}} \in\left(0, u_{\max }\right], u_{\mathrm{e}} \in\left[0, u_{\max }\right]$, and the steady-state equations:

$$
\begin{aligned}
& u_{\mathrm{f}}=U_{\mathrm{f}}(S), \\
& L(S)=\left(Y S_{\mathrm{in}}+u_{\mathrm{in}}\right) Q \\
& \Phi_{\mathrm{f}}=\widetilde{\Phi_{\mathrm{f}}}\left(U_{\mathrm{f}}(S)\right) \\
& u_{\mathrm{u}}=\frac{\min \left(\Phi_{\mathrm{f}}, \Phi_{\lim }\left(u_{\mathrm{f}},(r+w) Q\right)\right)}{(r+w) Q} \\
& u_{\mathrm{e}}=\frac{\Phi_{\mathrm{f}}-\min \left(\Phi_{\mathrm{f}}, \Phi_{\lim }\left(u_{\mathrm{f}},(r+w) Q\right)\right)}{(1-w) Q} .
\end{aligned}
$$

\section{Steady states with a CRitically LOADED SETtler}

In a given normal steady state of the ASP, the settler is either under-, over- or critically loaded. Which of these states is present can be checked by computing the excess flux:

$$
\begin{gathered}
E\left(u_{\mathrm{f}}, r, w\right):=\widetilde{\Phi_{\mathrm{f}}}\left(u_{\mathrm{f}}, r, Q\right)-\Phi_{\lim }\left(u_{\mathrm{f}},(r+w) Q\right)=(1+r) Q u_{\mathrm{f}}-\Phi_{\lim }\left(u_{\mathrm{f}},(r+w) Q\right) \\
=(1+r) Q u_{\mathrm{f}}-\min _{u_{\mathrm{f}} \leq v \leq u_{\max }}\left(A f_{\mathrm{b}}(v)+(r+w) Q v\right) \\
= \begin{cases}-A g\left(u_{\mathrm{f}},(1-w) Q\right), & u_{\mathrm{f}} \in\left[0, u_{\mathrm{m}}\left(Q_{\mathrm{u}}\right)\right] \cup\left[u_{\mathrm{M}}\left(Q_{\mathrm{u}}\right), u_{\max }\right], \\
(1+r) Q u_{\mathrm{f}}-A f_{\mathrm{b}}\left(u_{\mathrm{M}}\left(Q_{\mathrm{u}}\right)\right)-Q_{\mathrm{u}} u_{\mathrm{M}}\left(Q_{\mathrm{u}}\right), & u_{\mathrm{f}} \in\left(u_{\mathrm{m}}\left(Q_{\mathrm{u}}\right), u_{\mathrm{M}}\left(Q_{\mathrm{u}}\right)\right) .\end{cases}
\end{gathered}
$$

By definition, the settler is under-, over or critically loaded if $E<0, E>0$ or $E=0$, respectively. A critically loaded settler cannot handle any higher feed flux $\Phi_{\mathrm{f}}$ without being overloaded.

There is a slight difference between the two concepts 'critically loaded' and 'optimal operation' (see Definition 2.1), where the former is the easiest to capture mathematically $(E=0)$, but the latter the most desired in the application. Optimal operation and $u_{\mathrm{f}} \leq u_{\mathrm{M}}$ imply a critically loaded settler. We refer to [16]-[20] 
for precise definitions, details and examples. To find appropriate steady states, we require that the settler should be critically loaded, i.e.

$$
E\left(u_{\mathrm{f}}, r, w\right)=0 \quad \Longleftrightarrow \quad \Phi_{\mathrm{f}}=\Phi_{\lim }\left(u_{\mathrm{f}},(r+w) Q\right)
$$

in addition to (18)-(22) for a normal solution. Therefore, we now consider $r$ and $w$ as two additional variables. Since there are 7 variables and 6 equations, we expect a one-parameter family of solutions. As we shall prove, the control variable $r$ can be used as this parameter. By using (23), we can simplify the left-hand side of (19), defined by (13):

$$
L(S)=Q U_{\mathrm{f}}(S)(\alpha(r, w)+b \tau)+Y Q S, \quad 0<S \leq S_{\mathrm{in}} .
$$

Because of the one-to-one correspondence (18) between $u_{\mathrm{f}}$ and $S$ we can consider Eq. (19) in terms of $u_{\mathrm{f}}$. Therefore, we define

$$
G\left(u_{\mathrm{f}}, r, w\right):=L\left(U_{\mathrm{f}}^{-1}\left(u_{\mathrm{f}}\right)\right)=Q u_{\mathrm{f}}(\alpha(r, w)+b \tau)+Y Q U_{\mathrm{f}}^{-1}\left(u_{\mathrm{f}}\right),
$$

and we have the following system of equations for a steady-state solution with a critically loaded settler:

$$
\begin{aligned}
& E\left(u_{\mathrm{f}}, r, w\right)=0, \\
& G\left(u_{\mathrm{f}}, r, w\right)=Q\left(Y S_{\mathrm{in}}+u_{\mathrm{in}}\right), \\
& S=U_{\mathrm{f}}^{-1}\left(u_{\mathrm{f}}\right), \\
& u_{\mathrm{u}}=\frac{1+r}{r+w} u_{\mathrm{f}}, \\
& u_{\mathrm{e}}=0 .
\end{aligned}
$$

We prove below that $(23)-(24)$ define a curve in the $\left(u_{\mathrm{f}}, r, w\right)$-space in a region $\Sigma$ :

$$
\begin{aligned}
\Sigma & :=\left\{\left(u_{\mathrm{f}}, r, w\right) \in \mathbb{R}^{3}: 0 \leq u_{\mathrm{f}} \leq u_{\max }, 0 \leq w<1,0<r \leq \frac{\overline{\bar{Q}}_{\mathrm{u}}}{Q}-w\right\}, \\
\Sigma_{r w} & :=\left\{(r, w) \in \mathbb{R}^{2}: 0 \leq w<1,0<r \leq \frac{\overline{\bar{Q}}_{\mathrm{u}}}{Q}-w\right\} .
\end{aligned}
$$

The set $\Sigma_{r w}$ is the orthogonal projection of $\Sigma$ onto the $r w$-plane. The function $E$ is defined and continuous in $\bar{\Sigma}$ (the closure of $\Sigma$ ). The function $G$ is defined and smooth in $\bar{\Sigma} \backslash\left\{\left(u_{\mathrm{f}}, 0,0\right)\right\}$. According to Theorem 3.1, Eq. (19) for $L(S)$ has a unique solution, which implies that, for every $(r, w) \in \Sigma_{r w}$, (24) has a unique solution which we denote by $u_{\mathrm{f}}^{G}(r, w)$. Similarly, [23, Lemma 3.1] guarantees that (23) has a unique solution $u_{\mathrm{f}, \lim }(r, w) \in\left(0, u_{\max }\right)$. Thus, Eqs (23) and (24) can formally be written as follows:

$$
\begin{array}{ll}
E\left(u_{\mathrm{f}}, r, w\right)=0 & \Longleftrightarrow u_{\mathrm{f}}=u_{\mathrm{f}, \lim }(r, w), \\
G\left(u_{\mathrm{f}}, r, w\right)=Q\left(Y S_{\mathrm{in}}+u_{\mathrm{in}}\right) & \Longleftrightarrow u_{\mathrm{f}}=u_{\mathrm{f}}^{G}(r, w),
\end{array}
$$

see Figures 2 and 3. Generally, neither of the functions $u_{\mathrm{f}}^{G}$ and $u_{\mathrm{f}, \lim }$ can be described in closed algebraic form. In the following theorem (which proof can be found in the Appendix), note that all prerequisites are given without any dependence on $r$ and $w$. 

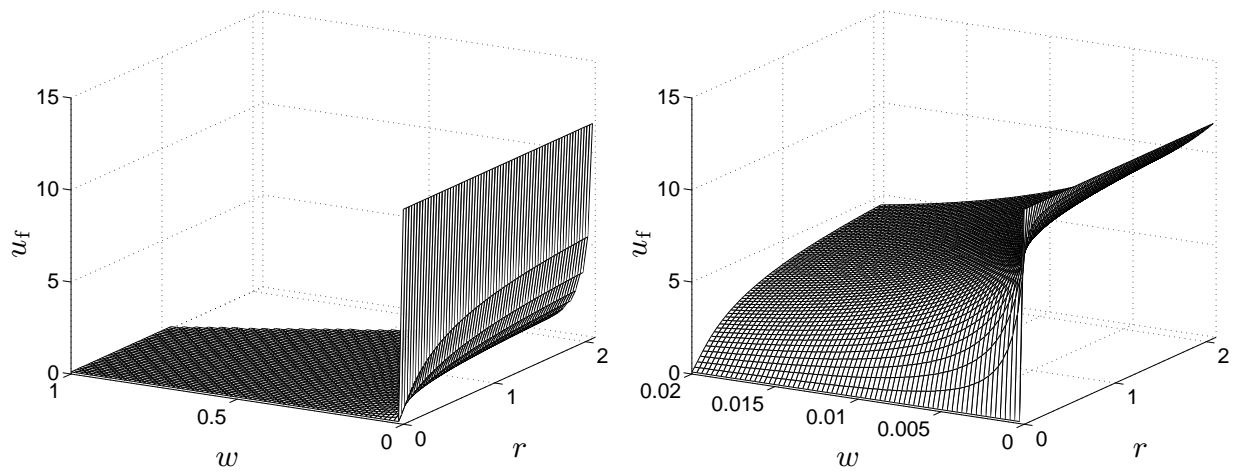

FiguRE 2. The graph of $u_{\mathrm{f}}=u_{\mathrm{f}}^{G}(r, w)$ with two scales for $w$.
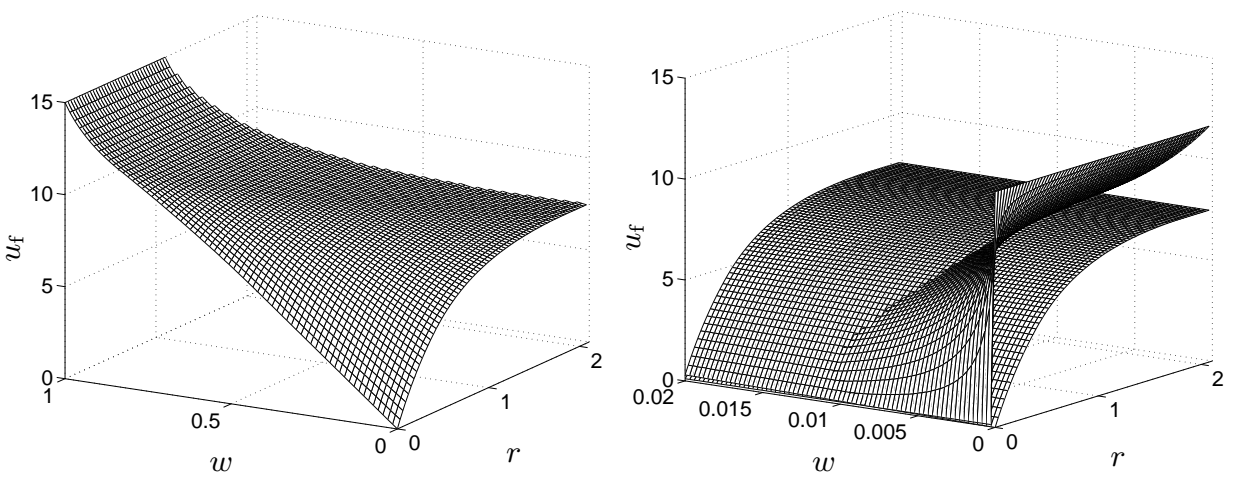

FiguRE 3. Left: The graph of $u_{\mathrm{f}}=u_{\mathrm{f}, \lim }(r, w)$. Right: The graphs of $u_{\mathrm{f}}^{G}$ and $u_{\mathrm{f}, \mathrm{lim}}$.

Theorem 4.1. Given fixed input variables $Q>0, S_{\mathrm{in}}>0$ and $u_{\mathrm{in}} \geq 0$. Assume that that the following inequalities hold:

$$
\begin{aligned}
v_{\mathrm{s}}(0) & >\frac{Q}{A}, \\
Y S_{\mathrm{in}}+u_{\text {in }} & \leq Y S_{\min }+u_{\max }(1+b \tau) .
\end{aligned}
$$

a. Assume that either $u_{\text {in }}>0$ holds, or $u_{\text {in }}=0$ and $\mu\left(S_{\text {in }}\right)>b$ hold, so that for every $(r, w) \in \Sigma_{r w}$ there exists a unique normal solution described by Theorem 3.1. Then Eqs (23) and (24) define uniquely a continuous curve

$$
\boldsymbol{\sigma}(r)=\left(u_{\mathrm{f}}^{\mathrm{CL}}(r), r, W(r)\right) \in \Sigma, \quad 0<r \leq r_{1},
$$

where $u_{\mathrm{f}}^{\mathrm{CL}}$ is a continuously increasing function and $W$ a continuous function, which satisfy $u_{\mathrm{f}}^{\mathrm{CL}}(0)=u_{\mathrm{f}}^{G}\left(0, w_{0}\right) \in\left(0, u_{\max }\right]$ for some $w_{0} \in(0,1]$ and $W(0)=w_{0}$. The curve $\boldsymbol{\sigma}$ may end in two qualitatively different boundary points of $\Sigma$; either $W\left(r_{1}\right)>0$ or $W\left(r_{1}\right)=0$ holds. If

$$
u_{\mathrm{f}, \lim }(r, 0)<u_{\mathrm{f}}^{G}(r, 0)=\sup _{(\rho, \omega) \in \Sigma_{r w}} u_{\mathrm{f}}^{G}(\rho, \omega)
$$


holds for all $r \in\left(0, \overline{\bar{Q}}_{\mathrm{u}} / Q\right]$, then $r_{1}+W\left(r_{1}\right)=\overline{\bar{Q}}_{\mathrm{u}} / Q$ holds; see Figure 4 . Otherwise, $r_{1}$ is equal to the supremum of the $r$ that satisfy (28); then $W\left(r_{1}\right)=0$ and $r_{1}<\bar{Q}_{\mathrm{u}} / Q$ hold. If

$$
v_{\mathrm{s}}\left(u_{\mathrm{inf}}\right)>\frac{Q}{A}
$$

holds (see Lemma A.3), then the functions $u_{\mathrm{f}}^{\mathrm{CL}}$ and $W$ are smooth. Otherwise they are smooth, except possibly at points $r_{0} \in\left(0, r_{1}\right)$ for which $u_{\mathrm{f}}^{\mathrm{CL}}\left(r_{0}\right)=u_{\mathrm{m}}\left(\left(r_{0}+W\left(r_{0}\right)\right) Q\right)$ holds.

b. Assume that $u_{\mathrm{in}}=0$ and $\mu\left(S_{\mathrm{in}}\right) \leq b$ hold. Then there exists no curve of the form (27).

Remark 4.1. Regarding (26) and (29), the example values (11) yield $v_{\mathrm{s}}(0) \approx$ $9.6 \mathrm{~m} / \mathrm{h}, v_{\mathrm{s}}\left(u_{\mathrm{infl}}\right) \approx 1.3 \mathrm{~m} / \mathrm{h}$ and $Q / A \approx 0.7 \mathrm{~m} / \mathrm{h}$.

Theorem 4.1 states how the control variables $r$ and $w$ should be adjusted to obtain a steady state of the ASP with a critically loaded settler. Note that the decreasing relation $u_{\mathrm{f}}=U_{\mathrm{f}}(S)$, see (18), means that the corresponding substrate values along the curve $\sigma$ decrease for increasing $r$; cf. Figure 5 (left). The curve $\sigma$ can be computed numerically in the following way. Solve for $w$ in Eq. (24) and substitute the expression (in $r$ and $u_{\mathrm{f}}$ ) into (23), which is then solved numerically for $u_{\mathrm{f}}$ for an increasing sequence of $r \geq 0$. Since we have an explicit representation $u_{\mathrm{f}}=U_{\mathrm{f}}(S)$, see (18), but maybe not its inverse, we prefer to express $w$ in terms of $S$ and $r$ first:

$$
\begin{aligned}
G\left(U_{\mathrm{f}}(S), r, w\right) & =Q\left(Y S_{\mathrm{in}}+u_{\mathrm{in}}\right) \quad \Longleftrightarrow \quad U_{\mathrm{f}}(S)(\alpha(r, w)+b \tau)+Y S=Y S_{\mathrm{in}}+u_{\mathrm{in}} \\
& \Longleftrightarrow \alpha(r, w)=\beta(S) \quad \Longleftrightarrow \quad w=\frac{\beta(S) r}{1-\beta(S)+r},
\end{aligned}
$$

where

$$
\beta(S):=\frac{Y\left(S_{\mathrm{in}}-S\right)+u_{\mathrm{in}}}{U_{\mathrm{f}}(S)}-b \tau=\left(\mu(S)\left(1+\frac{u_{\mathrm{in}}}{Y\left(S_{\mathrm{in}}-S\right)}\right)-b\right) \tau .
$$
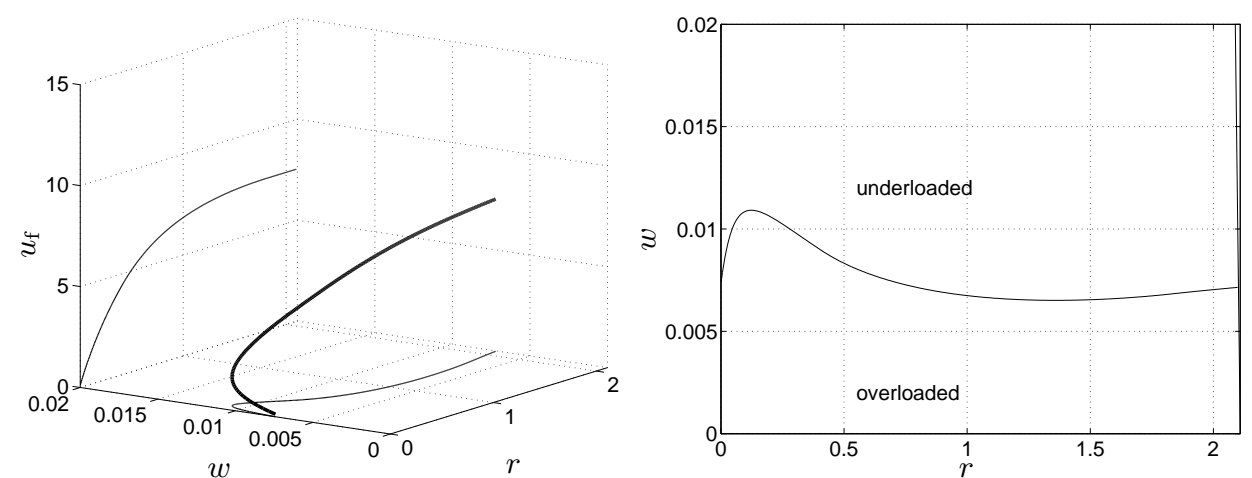

Figure 4. Left: The curve $\boldsymbol{\sigma}(r)$ and its projection onto the $r w$ and $r u_{\mathrm{f}}$-planes. Compare with Figure 3 (right). Right: The projection of the curve onto the $r w$-plane. The inclined straight line on the right has the equation $(r+w) Q=\overline{\bar{Q}}_{\mathrm{u}}$. 

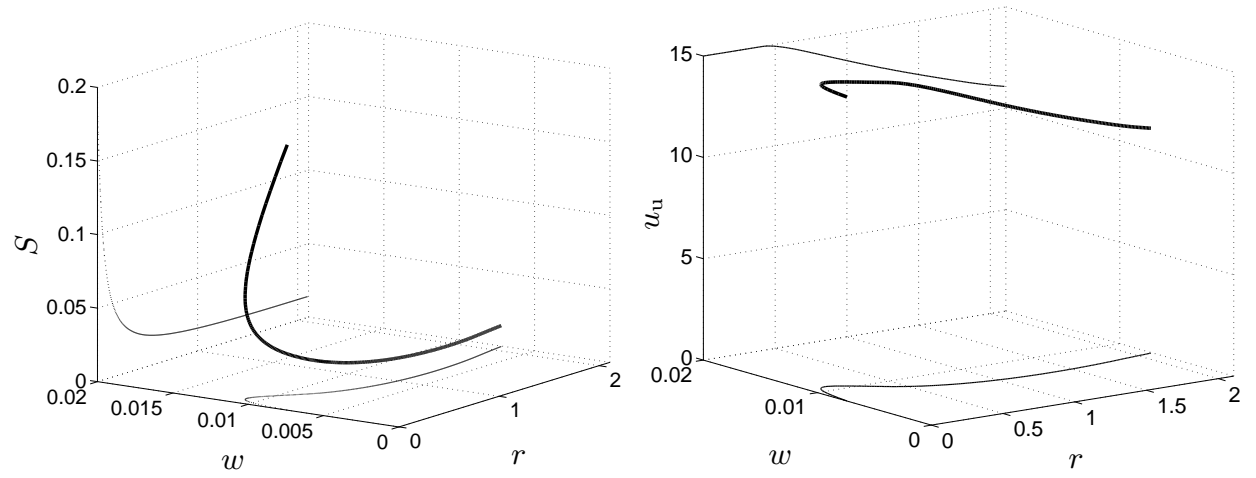

Figure 5. Left: The curve $\boldsymbol{\sigma}^{S}(r)=\left(S^{\mathrm{CL}}(r), r, W(r)\right)$. Note that $S^{\mathrm{CL}}$ is decreasing. Right: The curve $\boldsymbol{\sigma}^{u_{\mathrm{u}}}(r)=\left(u_{\mathrm{u}}^{\mathrm{CL}}(r), r, W(r)\right)$. For $r \leq 0.4, u_{\mathrm{u}}^{\mathrm{CL}}(r)=u_{\max }=15 \mathrm{~kg} / \mathrm{m}^{3}$ holds. Note that the projections of these curves onto the rw-plane coincide with the one in Figure 4 (right).

Then, for each $r$,

$$
E\left(U_{\mathrm{f}}(S), r, \frac{\beta(S) r}{1-\beta(S)+r}\right)=0
$$

is solved for $S$. This defines a function $S^{\mathrm{CL}}(r)$. Setting

$$
W(r):=\frac{\beta\left(S^{\mathrm{CL}}(r)\right) r}{1-\beta\left(S^{\mathrm{CL}}(r)\right)+r},
$$

the curve is $\boldsymbol{\sigma}(r)=\left(U_{\mathrm{f}}\left(S^{\mathrm{CL}}(r)\right), r, W(r)\right)$; see Figure 4. Of additional interest are the curves $\boldsymbol{\sigma}^{S}(r):=\left(S^{\mathrm{CL}}(r), r, W(r)\right)$ and $\boldsymbol{\sigma}^{u_{\mathrm{u}}}(r):=\left(u_{\mathrm{u}}^{\mathrm{CL}}(r), r, W(r)\right):=$ $\left(\frac{1+r}{r+W(r)} u_{\mathrm{f}}(r), r, W(r)\right)$ (cf. $\left.(25)\right)$, which are plotted in Figure 5. All three curves $\boldsymbol{\sigma}, \boldsymbol{\sigma}^{S}$ and $\boldsymbol{\sigma}^{u_{\mathrm{u}}}$ have the same projection onto the $r w$-plane; see Figure 4 (right).

The region above/below the curve in the $r w$-plane in Figure 4 (right) can be denoted the underloaded/overloaded region for the following reason. Given a point $\left(r_{0}, w_{0}\right)$ on the curve, where $E\left(u_{\mathrm{f}}^{G}\left(r_{0}, w_{0}\right), r_{0}, w_{0}\right)=0$. We get

$$
\frac{\partial}{\partial w} E\left(u_{\mathrm{f}}^{G}\left(r_{0}, w_{0}\right), r_{0}, w_{0}\right)=\frac{\partial E}{\partial u_{\mathrm{f}}} \frac{\partial u_{\mathrm{f}}^{G}}{\partial w}+\frac{\partial E}{\partial w}<0
$$

because of Lemma A.2. Hence, $E\left(u_{\mathrm{f}}^{G}\left(r_{0}, w_{1}\right), r_{0}, w_{1}\right)<0$ holds for small $w_{1}>$ $w_{0}$, i.e., the settler is underloaded. This holds for the whole region above the control curve because of the uniqueness in Theorem 4.1. Analogously, the settler is overloaded below the curve.

\section{Manual CONTROL}

5.1. Dependencies of desired steady states on input variables. It is of interest to analyze how the curve $\boldsymbol{\sigma}$ varies with the input parameters in steady state, i.e., we would like to investigate

$$
\boldsymbol{\sigma}\left(r, S_{\mathrm{in}}, u_{\mathrm{in}}, Q\right)=\left(u_{\mathrm{f}}^{\mathrm{CL}}\left(r, S_{\mathrm{in}}, u_{\mathrm{in}}, Q\right), r, W\left(r, S_{\mathrm{in}}, u_{\mathrm{in}}, Q\right)\right) .
$$




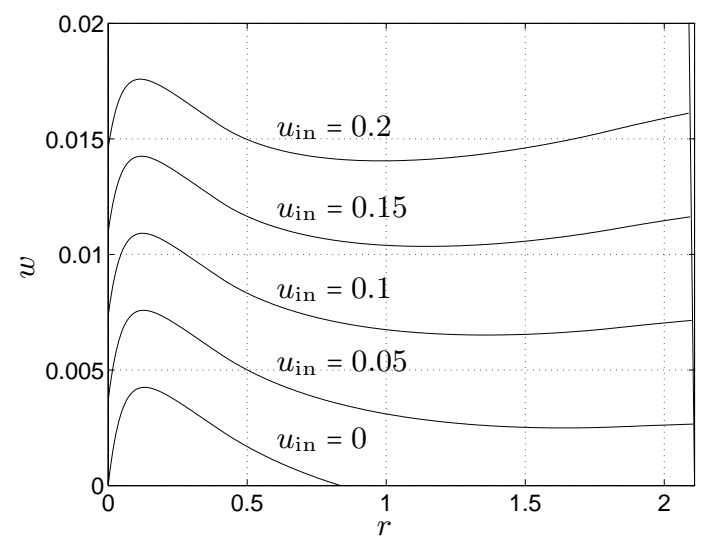

Figure 6. Projection of $\boldsymbol{\sigma}$-curves onto the $r w$-plane for $S_{\text {in }}=$ $0.2 \mathrm{~kg} / \mathrm{m}^{3}, Q=1000 \mathrm{~m}^{3} / \mathrm{h}$ and different values of $u_{\text {in }}\left[\mathrm{kg} / \mathrm{m}^{3}\right]$.

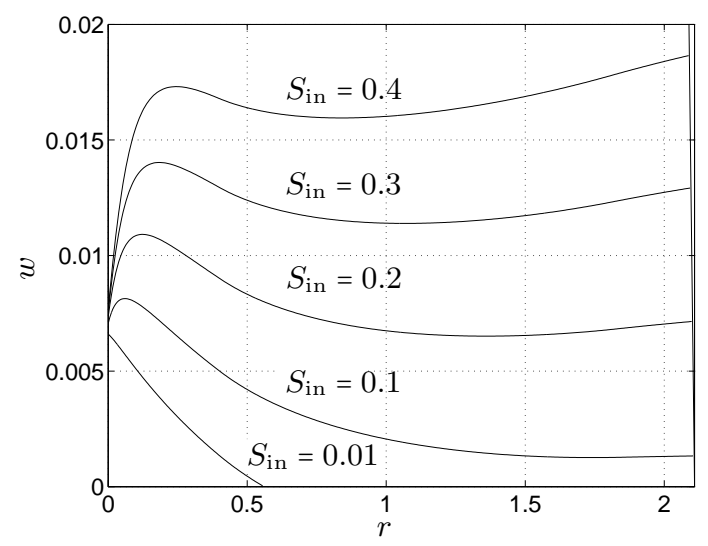

Figure 7. Projection of $\sigma$-curves onto the $r w$-plane for $u_{\text {in }}=$ $0.1 \mathrm{~kg} / \mathrm{m}^{3}, Q=1000 \mathrm{~m}^{3} / \mathrm{h}$ and different values of $S_{\mathrm{in}}\left[\mathrm{kg} / \mathrm{m}^{3}\right]$ (left).

It turns out that there are certain monotonicity properties with respect to $S_{\text {in }}$ and $u_{\text {in }}$; see the following theorem and Figures 6-7, whereas the dependency on $Q$ is more complex; see Figure 8.

Theorem 5.1. Given a curve

$$
\boldsymbol{\sigma}\left(r, S_{\mathrm{in}}, u_{\mathrm{in}}\right)=\left(u_{\mathrm{f}}^{\mathrm{CL}}\left(r, S_{\mathrm{in}}, u_{\mathrm{in}}\right), r, W\left(r, S_{\mathrm{in}}, u_{\mathrm{in}}\right)\right)
$$

in Theorem 4.1 and the belonging $S^{\mathrm{CL}}\left(r, S_{\mathrm{in}}, u_{\mathrm{in}}\right)$. The following monotonicity properties hold for every fixed $r>0$ and corresponding point on the curve, where $\Delta S_{\mathrm{in}}$ denotes a small change in $S_{\mathrm{in}}$, etc.:

$$
\frac{\Delta u_{\mathrm{f}}^{\mathrm{CL}}}{\Delta S_{\text {in }}}>0, \quad \frac{\Delta W}{\Delta S_{\text {in }}}>0, \quad \frac{\Delta S^{\mathrm{CL}}}{\Delta S_{\text {in }}}>0, \quad \frac{\Delta u_{\mathrm{f}}^{\mathrm{CL}}}{\Delta u_{\text {in }}}>0, \quad \frac{\Delta W}{\Delta u_{\text {in }}}>0, \quad \frac{\Delta S^{\mathrm{CL}}}{\Delta u_{\text {in }}}<0 .
$$

The proof of this theorem can be found in the Appendix. The following interpretation can be made. Given an ASP in steady state with a critically loaded 


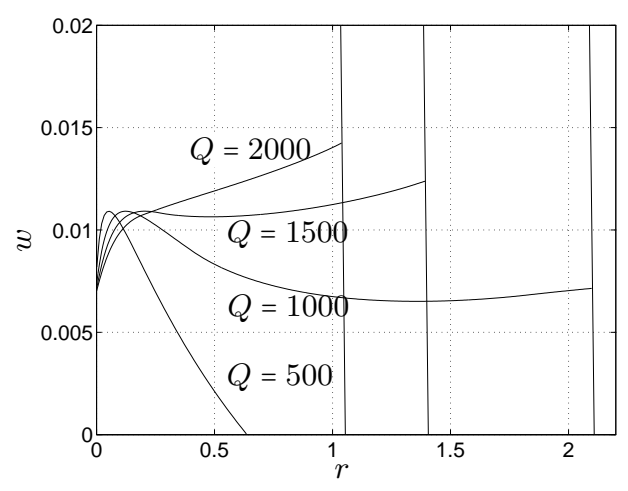

Figure 8. Curves for $S_{\text {in }}=0.2 \mathrm{~kg} / \mathrm{m}^{3}, u_{\text {in }}=0.1 \mathrm{~kg} / \mathrm{m}^{3}$ and different values of $Q\left[\mathrm{~m}^{3} / \mathrm{h}\right]$. Note that the (almost vertical) lines $(r+w) Q=\overline{\bar{Q}}_{\mathrm{u}}$ depend on $Q$.

settler. If $S_{\text {in }}$ is increased to a new constant value, a new steady state will appear with an overloaded settler, since the curve (in the $r w$-plane) lies above the present point $(r, w)$. To get a new steady state with a critically loaded settler, $w$ has to be increased to $w_{1}$ so that the curve passes through $\left(r, w_{1}\right)$. According to the theorem, the new steady-state substrate concentration is higher than the one before.

5.2. Demonstration of manual control of a load disturbance in $S_{\mathrm{in}}$. The numerical simulations presented in this article have been performed on a fixed grid in time and space. Explicit Euler time steps are used and they are bounded by the spatial grid size via the standard CFL condition for the hyperbolic PDEs (4)(5). The fluxes of these PDEs are approximated by the consistent Enguist-Osher numerical flux, adapted to handle the spatial discontinuities; see [8]. The height of the clarification zone has been set to $H=1 \mathrm{~m}$ and depth of the thickening zone to $B=3 \mathrm{~m}$. All simulations start with an ASP in a steady state with a settler in optimal operation with a sludge blanket at $x_{\mathrm{sb}}=1.7 \mathrm{~m}$. The example parameter values (11) are used initially except for the control variables.

With the simulation in Figure 9 we demonstrate how manual control with the result of Theorem 3.1 can be used to obtain a steady-state solution with a sludge blanket in the thickening zone. Initially, the control curve is the one in Figure 4 (right), and it can also be found in Figures $6\left(S_{\text {in }}=0.2 \mathrm{~kg} / \mathrm{m}^{3}\right), 7\left(u_{\text {in }}=0.1 \mathrm{~kg} / \mathrm{m}^{3}\right)$ and $8\left(Q=1000 \mathrm{~m}^{3} / \mathrm{h}\right)$. We choose a reference value for the substrate concentration: $S^{\text {ref }}=0.023 \mathrm{~kg} / \mathrm{m}^{3}$. The corresponding (uniquely determined) point on the control curve is $(r, w) \approx(0.44,0.0087)$. As the simulation shows, the ASP is in steady state during the first 20 hours. At $t=20 \mathrm{~h}$, the incoming substrate concentration makes a jump up to $S_{\text {in }}=0.3 \mathrm{~kg} / \mathrm{m}^{3}$. Although the concentrations in the biological reactor fairly quickly converge to new constant values, the sludge blanket rises slowly. The settler is going to be overloaded, which can also be inferred from Figure 7; the point $(r, w) \approx(0.44,0.0087)$ lies below the curve with $S_{\text {in }}=0.3 \mathrm{~kg} / \mathrm{m}^{3}$. To prevent an overflow and to ensure that the reference value of the substrate concentration is regained, a manual control is made at $t=200 \mathrm{~h}$ : the control parameters are set to $(r, w) \approx(1.27,0.011)$, which is the point on the new control curve where $S^{\mathrm{CL}}=S^{\mathrm{ref}}$. 

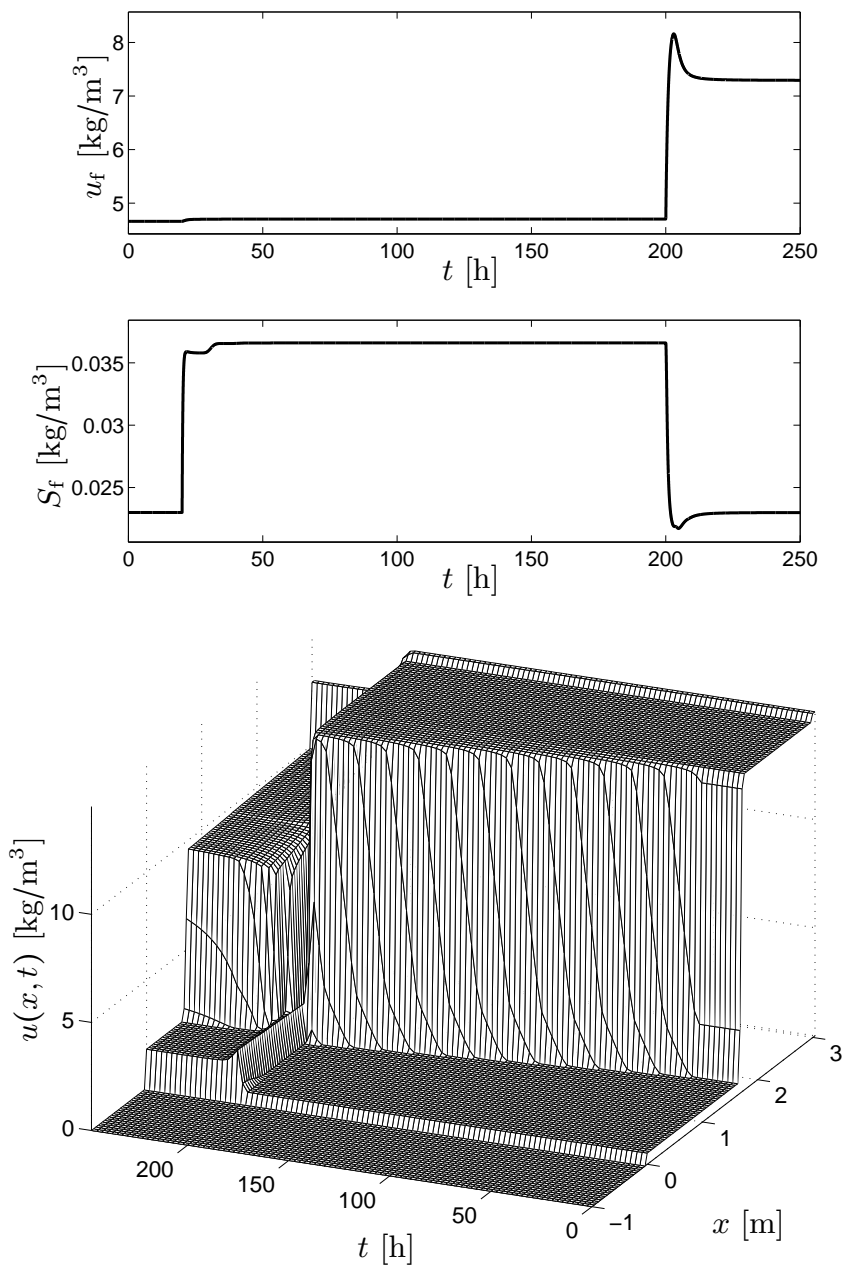

Figure 9. A dynamic simulation with a step increase in $S_{\text {in }}(t)$ at $t=20 \mathrm{~h}$ and a manual control action at $t=200 \mathrm{~h}$. The threedimensional graph shows the biomass concentration in the settler.

Accordingly, the simulation shows that a new steady state arises with a stationary sludge blanket and with the desired $S_{\mathrm{f}}(t) \rightarrow S^{\mathrm{ref}}=0.023 \mathrm{~kg} / \mathrm{m}^{3}$ as $t \rightarrow \infty$. Of course, the effluent concentration $S_{\mathrm{e}}(t)$ also converges, since this is $S_{\mathrm{f}}(t)$ time delayed.

\section{Automatic control}

Automatic control is possible to satisfy the control objective for the ASP. The idea is the following. Given $S^{\text {ref }}$, define reference values $r^{\text {ref }}$ and $w^{\text {ref }}$ in accordance with Theorem 4.1. Setting $(r(t), w(t)) \equiv\left(r^{\mathrm{ref}}, w^{\mathrm{ref}}\right)$ we get a critically loaded settler in steady state; however, generally not with a sludge blanket at the desired location $x_{\mathrm{sb}}^{\mathrm{ref}}$. For an uncoupled settler, the sludge blanket level $x_{\mathrm{sb}}$ can be controlled to $x_{\mathrm{sb}}^{\mathrm{ref}}$ with the proportional regulator in [20], which controls the mass in the settler by adjusting the volumetric flow rate $Q_{\mathrm{u}}$. This works because of an affine relationship between the steady-state mass and the sludge blanket level for a critically loaded 
settler. Since $Q_{\mathrm{u}}=(r+w) Q$, we can write the regulator in [20] on the following form expressed by means of our control variables $\left(K_{\mathrm{P}}^{m}>0\right)$ :

$$
(r(t)+w(t)) Q=\left(r^{\mathrm{ref}}+w^{\mathrm{ref}}\right) Q+K_{\mathrm{P}}^{m}\left(m(t)-m^{\mathrm{ref}}\right),
$$

where the reference mass $m^{\text {ref }}$ is a decreasing affine function of $x_{\mathrm{sb}}^{\text {ref }}$ :

$$
m^{\mathrm{ref}}:=A\left(B u_{\mathrm{M}}\left(Q_{\mathrm{u}}^{\mathrm{ref}}\right)-x_{\mathrm{sb}}^{\mathrm{ref}}\left(u_{\mathrm{M}}\left(Q_{\mathrm{u}}^{\mathrm{ref}}\right)-u_{\mathrm{m}}\left(Q_{\mathrm{u}}^{\mathrm{ref}}\right)\right)\right),
$$

and where $Q_{\mathrm{u}}^{\mathrm{ref}}:=\left(r^{\mathrm{ref}}+w^{\mathrm{ref}}\right) Q$. Note that (33) is the steady-state relationship between the mass and the sludge blanket for a settler in optimal operation. The regulator (32) does not require any measurement of the sludge blanket level $x_{\mathrm{sb}}(t)$, but requires that the inlet and outlet concentrations $u_{\mathrm{f}}(t), u_{\mathrm{e}}(t)$ and $u_{\mathrm{u}}(t)$ are measured so that the total mass in the settler is known:

$$
m(t)=m_{0}+\int_{0}^{t}\left(Q_{\mathrm{f}}(s) u_{\mathrm{f}}(s)-Q_{\mathrm{u}}(s) u_{\mathrm{u}}(s)-Q_{\mathrm{e}}(s) u_{\mathrm{e}}(s)\right) d s, \quad t>0,
$$

where $m_{0}$ is the initial mass at $t=0$. Despite the fact that the regulator (32) is proportional with respect to the mass deviation, $x_{\mathrm{sb}}(t) \rightarrow x_{\mathrm{sb}}^{\mathrm{ref}}$ as $t \rightarrow \infty$ occurs when $Q_{\mathrm{u}}$ is used as the control variable for an uncoupled settler (under the assumption that convergence to a steady-state solution occurs); see [20, Theorem 4.1]. The result holds for a given fixed feed volumetric flow $Q_{\mathrm{f}}$.

For the ASP, the variable $Q_{\mathrm{f}}$ depends on one of the control variables: $Q_{\mathrm{f}}=$ $(1+r) Q$. Nevertheless, it is possible to choose $w(t) \equiv w^{\text {ref }}$ and let $(32)$ define the control variable $r(t)$. Alternatively, one may set $r(t) \equiv r^{\text {ref }}$ and let (32) define the control variable $w(t)$. Since there are two control variables, a more general approach is to utilize the deviation $S_{\mathrm{f}}(t)-S^{\mathrm{ref}}$ in the controller; see (36) below. According to the control objective in Definition 2.2, $S_{\mathrm{e}}(t)$ should lie close to $S^{\text {ref }}$, but note that $S_{\mathrm{e}}(t)$ is a mere time delay of $S_{\mathrm{f}}(t)$, wherefore $S_{\mathrm{f}}(t)$ is the better variable to control. The fact that the constant $K_{\mathrm{P}}^{S}$ in (36) should be nonnegative can be inferred from the results of [23], from which one can conclude that, given a normal steady-state solution, a step increase of $r$ will result in a decrease in $S_{\mathrm{f}}(t)$.

6.1. A control algorithm. In the following algorithm; see Figure 10, we assume that $S^{\mathrm{ref}}, x_{\mathrm{sb}}^{\mathrm{ref}}$ and the inputs $Q, u_{\mathrm{in}}$ and $S_{\mathrm{in}}$ are known and that $S_{\mathrm{f}}, u_{\mathrm{f}}, u_{\mathrm{e}}$ and $u_{\mathrm{u}}$ are measured (we do not write out the time dependencies). The state variable $m$ is defined via (cf. (34)):

$$
\frac{d m}{d t}=Q\left((1+r) u_{\mathrm{f}}-(r+w) u_{\mathrm{u}}-(1-w) u_{\mathrm{e}}\right) .
$$

Do the following steps at time $t$ :

1. Let $r^{\text {ref }}$ be the unique solution of (31) with $S=S^{\text {ref }}$ and define $w^{\text {ref }}$ by (30).

2. Set $Q_{\mathrm{u}}^{\text {ref }}:=\left(r^{\text {ref }}+w^{\text {ref }}\right) Q$, define $u_{\mathrm{M}}\left(Q_{\mathrm{u}}^{\text {ref }}\right)$ and $u_{\mathrm{m}}\left(Q_{\mathrm{u}}^{\text {ref }}\right)$ by $(9)$ and $(10)$, and define the reference mass $m^{\text {ref }}$ by (33).

3. For constants $K_{\mathrm{P}}^{m}>0, K_{\mathrm{P}}^{S} \geq 0$, define

$$
\begin{aligned}
r & :=r^{\mathrm{ref}}+K_{\mathrm{P}}^{S}\left(S_{\mathrm{f}}-S^{\mathrm{ref}}\right), \\
w & :=w^{\mathrm{ref}}+\frac{K_{\mathrm{P}}^{m}}{Q}\left(m-m^{\mathrm{ref}}\right)-K_{\mathrm{P}}^{S}\left(S_{\mathrm{f}}-S^{\mathrm{ref}}\right) .
\end{aligned}
$$




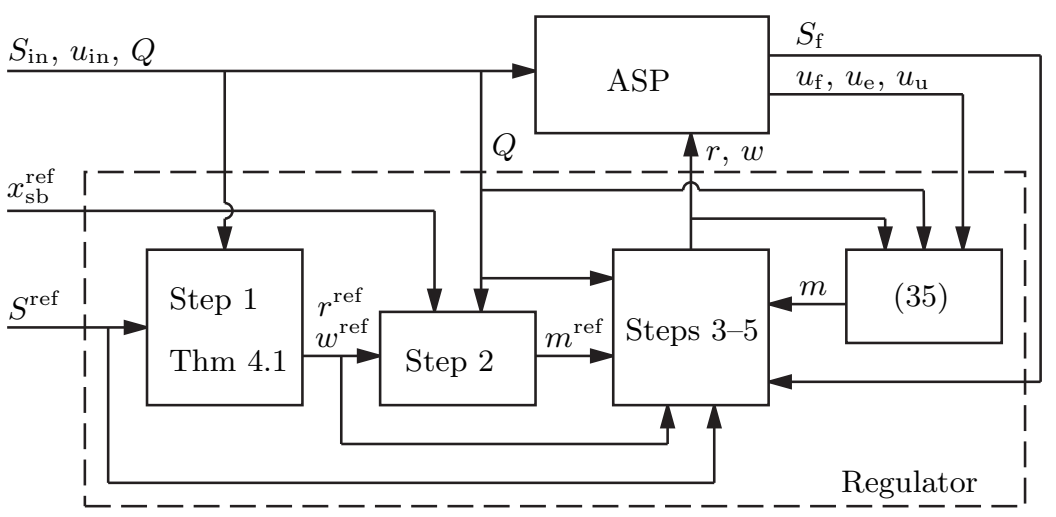

Figure 10. The closed-loop system.

4. Redefine, in the following order, the control variables with respect to saturating bounds:

$$
\begin{aligned}
w & :=\max \left(0, \min \left(w_{\max }, w\right)\right), \\
r & :=-w+\frac{1}{Q} \max \left(Q_{\mathrm{u}}^{\min }, \min \left(Q_{\mathrm{u}}^{\max },(r+w) Q\right)\right) .
\end{aligned}
$$

for some given $0<w_{\max } \leq 1, Q_{\mathrm{u}}^{\max }>0$ and $Q_{\mathrm{u}}^{\min } \geq 0$.

The saturating bounds $w_{\max }$ and $Q_{\mathrm{u}}^{\max }$ can be set, for instance, because of reduced pump capacities. At least, $Q_{\mathrm{u}}^{\max }$ should be less than $\overline{\bar{Q}}_{\mathrm{u}}$; see Definition 2.1. If the control objective contains the requirement that the underflow concentration $u_{\mathrm{u}}(t)$ (during dynamic operation) should be greater than a pre-specified bound, then it is possible to choose $Q_{\mathrm{u}}^{\max }$ so that this is satisfied as long as the sludge blanket level does not reach the bottom; see [18, Theorem 4.1]. To prevent an overflow situation, the minimum bound $Q_{\mathrm{u}}^{\min }(t)$ can be defined as a function of $u_{\mathrm{f}}(t)$ and $\max _{t \geq 0} Q_{\mathrm{f}}(t)=(1+r(t)) Q(t)$; see [19, Theorem 2.1] and [20]. In all simulations that will follow, only the lower bound 0 for $w$ in (38) will be invoked.

6.2. On the closed-loop performance and stability. The dynamics of the open-loop ODE-PDE system (2)-(5) is difficult to analyze. In fact, the classification of all steady states turned out to be rather complex; see [23]. However, a stability analysis of each steady state could be performed for small times after a disturbance, also a large such. The closed-loop system, when the regulator equations (36)-(37) are added, is also difficult to analyze generally, but we have the following results. We use the notation $r(\infty):=\lim _{t \rightarrow \infty} r(t)$.

Theorem 6.1. Given constant inputs $S_{\mathrm{in}}, u_{\mathrm{in}}, Q$ and constant reference values $S^{\mathrm{ref}}$ and $x_{\mathrm{sb}}^{\mathrm{ref}}$ that satisfy the requirements of Theorem 4.1; assume that the closed-loop system (2)-(5), (36)-(37) has a solution such that the settler is in optimal operation for all $t$ and that it converges to a steady state as $t \rightarrow \infty$ with $u_{\mathrm{f}}(\infty) \leq u_{\mathrm{M}}\left(Q_{\mathrm{u}}(\infty)\right)$. Then $S_{\mathrm{f}}(t) \rightarrow S^{\mathrm{ref}}$ and $x_{\mathrm{sb}}(t) \rightarrow x_{\mathrm{sb}}^{\mathrm{ref}}$ as $t \rightarrow \infty$.

Proof. The condition $u_{\mathrm{f}}(\infty) \leq u_{\mathrm{M}}\left(Q_{\mathrm{u}}(\infty)\right)$ implies that the settler is critically loaded in steady state; see [17, Section 3], with $x_{\mathrm{sb}}(\infty) \in[0, B]$. Then both 
$\left(u_{\mathrm{f}}(\infty), r(\infty), w(\infty)\right)$ and $\left(u_{\mathrm{f}}^{\mathrm{CL}}\left(r^{\mathrm{ref}}\right), r^{\mathrm{ref}}, w^{\mathrm{ref}}\right)$ are points on the curve $\boldsymbol{\sigma}$ in Theorem 4.1. The regulator (36)-(37) yields

$$
\begin{aligned}
r(\infty) & =r^{\mathrm{ref}}+K_{\mathrm{P}}^{S}\left(S_{\mathrm{f}}(\infty)-S^{\mathrm{ref}}\right), \\
w(\infty) & =w^{\mathrm{ref}}+\frac{K_{\mathrm{P}}^{m}}{Q}\left(m(\infty)-m^{\mathrm{ref}}(\infty)\right)-K_{\mathrm{P}}^{S}\left(S_{\mathrm{f}}(\infty)-S^{\mathrm{ref}}\right) .
\end{aligned}
$$

If $K_{\mathrm{P}}^{S}=0$, then $r(\infty)=r^{\text {ref }}$ holds, and according to Theorem 4.1 this $r$-value corresponds to a unique point on the $\boldsymbol{\sigma}$-curve, so that $w(\infty)=w^{\text {ref }}$ and $S_{\mathrm{f}}(\infty)=S^{\text {ref }}$ hold. Eq. (41) then gives $m(\infty)=m^{\text {ref }}$, which in turn, via (33) yields $x_{\mathrm{sb}}(\infty)=x_{\mathrm{sb}}^{\mathrm{ref}}$. Consider the case when $K_{\mathrm{P}}^{S}>0$. Assume that $r(\infty)<r^{\text {ref }}$. Since $u_{\mathrm{f}}^{\mathrm{CL}}$ is increasing by Theorem 4.1, $u_{\mathrm{f}}^{\mathrm{CL}}(r(\infty))<u_{\mathrm{f}}^{\mathrm{CL}}\left(r^{\mathrm{ref}}\right)$ holds. The decreasing function $U_{\mathrm{f}}^{-1}$ (see (15)) then implies $S_{\mathrm{f}}(\infty)>S^{\text {ref }}$. The latter inequality and (40) imply $r(\infty)>r^{\text {ref }}$, which is a contradiction. Starting with $r(\infty)>r^{\text {ref }}$ also yields a contradiction. Hence, $r(\infty)=r^{\text {ref }}$ holds, and we can make the same conclusion as above.

We shall now investigate the local stability for small times of the closed-loop system near a steady state where the settler is in optimal operation. We will then utilize the ODEs (2)-(3) and (35), which we rewrite as:

$$
\begin{aligned}
\tau \frac{d S_{\mathrm{f}}}{d t} & =S_{\mathrm{in}}+r S_{\mathrm{u}}-(1+r) S_{\mathrm{f}}-\tau \frac{\mu\left(S_{\mathrm{f}}\right)}{Y} u_{\mathrm{f}}, \\
\tau \frac{d u_{\mathrm{f}}}{d t} & =u_{\mathrm{in}}+r u_{\mathrm{u}}-(1+r) u_{\mathrm{f}}+\tau\left(\mu\left(S_{\mathrm{f}}\right)-b\right) u_{\mathrm{f}}, \\
\tau \frac{d m}{d t} & =V(1+r) u_{\mathrm{f}}-V(r+w) u_{\mathrm{u}}-V(1-w) u_{\mathrm{e}},
\end{aligned}
$$

where $r$ and $w$ are given by (36)-(37). The underflow variables $S_{\mathrm{u}}$ and $u_{\mathrm{u}}$ in (42) are "outputs" from the settler PDEs (4)-(5). The solutions of such hyperbolic PDEs have finite signal speeds, which means that after a change at the inlet, there is a time period during which this disturbance propagates through the thickening zone to the underflow discharge. During this time period, the underflow concentrations $S_{\mathrm{u}}$ and $u_{\mathrm{u}}$ are independent of any disturbances in the feed variables. This fact can be utilized to prove the following theorem. The proof can be found in the Appendix and the idea is that the qualitative behaviour of the closed-loop system is governed by the Jacobian of (42).

Theorem 6.2. The closed-loop system described by (42), (36)-(37) and the PDEs (4)-(5) is, at least during a finite time, locally stable in a normal steady state with the settler in optimal operation with respect to small disturbances in $S_{\mathrm{f}}, u_{\mathrm{f}}, m$ and $u(x, 0), 0<x<B$.

We shall below investigate different kinds of disturbances and thereby give guidelines for the tuning of the regulator. Large values of $K_{\mathrm{P}}^{m}$ or $K_{\mathrm{P}}^{S}$ increase the likelihood of reaching the saturating bounds in (38)-(39). Small values of these parameters imply small variations in the volumetric flows in the plant, which are desired for different reasons, such as economic or avoidance of turbulence. The proof of Theorem 6.2 yields that only one of the eigenvalues of the Jacobian of (42), the one connected to the mass variable, contains $K_{\mathrm{P}}^{m}$. This indicates that a higher value of $K_{\mathrm{P}}^{m}$ will lead to a faster control of the mass. However, the sludge blanket moves slowly for any value of $K_{\mathrm{P}}^{m}$. This has been demonstrated for an 
uncoupled settler in [20, Figs. 11, 12] and we shall see that this holds for the ASP as well. We therefore expect that a small value of $K_{\mathrm{P}}^{m}$ is appropriate.

The dynamics of the biological reactor is much faster than the settler, and we expect a fast convergence of the reactor concentrations $S_{\mathrm{f}}$ and $u_{\mathrm{f}}$ after a disturbance even for small values of $K_{\mathrm{P}}^{S}$. Note that Theorems 6.1 and 6.2 hold also for $K_{\mathrm{P}}^{S}=0$.

6.3. Tracking reference values. In all simulations, we start in the same normal steady state as in Section 5.2. We investigate how the regulator in Section 6.1 can track the reference values by letting $x_{\mathrm{sb}}^{\mathrm{ref}}(t)$ make a step decrease from 1.7 to $1.2 \mathrm{~m}$ at $t=5 \mathrm{~h}$ and $S^{\text {ref }}(t)$ a step decrease from 0.023 to $0.015 \mathrm{~kg} / \mathrm{m}^{3}$ at $t=100 \mathrm{~h}$. In Figures 11 and 12, simulations with different values of $K_{\mathrm{P}}^{m}$ and $K_{\mathrm{P}}^{S}$ are shown. For comparison, the case when the regulator is not connected $\left(K_{\mathrm{P}}^{m}=K_{\mathrm{P}}^{S}=0\right)$ is contained in Figure 11. Note that in accordance with the findings in Sections 5 and 6.2 , convergence of the reactor concentrations occur fast even without the regulator, however, at least a small value on $K_{\mathrm{P}}^{m}$ is needed to adjust the sludge blanket level. Figure 11 gives that $K_{\mathrm{P}}^{m}=0.01$ means a faster convergence of $x_{\mathrm{sb}}$ in comparison to $K_{\mathrm{P}}^{m}=0.001$; however, to the cost of a much higher $w$, which might not be desirable. The remedy is to limit $w$ by setting an appropriate value on $w_{\max }$ in (38). Thus, we have chosen $K_{\mathrm{P}}^{m}=0.01$ for the simulations in Figure 12. These results imply that $K_{\mathrm{P}}^{S}=0$ is sufficient for tracking reference values.

6.4. Load disturbances. We apply the following load disturbances: at $t=5 \mathrm{~h}$, $S_{\text {in }}(t)$ is increased from 0.2 to $0.3 \mathrm{~kg} / \mathrm{m}^{3}$ and at $t=20 \mathrm{~h}, Q(t)$ is decreased from 1000 to $800 \mathrm{~m}^{3} / \mathrm{h}$; see Figures 13 and 14 . As in Section 6.3 , we conclude that $K_{\mathrm{P}}^{S}=0$ and $K_{\mathrm{P}}^{m}=0.01$ are suitable values which we keep in the next subsection.

6.5. Other disturbances. An advantage of the conclusion above that $K_{\mathrm{P}}^{S}=0$ can be used, is that the output $S_{\mathrm{f}}$ need not be measured. Recall that the sludge blanket level $x_{\mathrm{sb}}$ need not be measured either. However; to handle unmeasured disturbances with nonzero mean values in the outputs $u_{\mathrm{f}}, u_{\mathrm{e}}, u_{\mathrm{u}}$ or the inputs $S_{\mathrm{in}}$, $u_{\text {in }}$, and $Q$, one has to measure $S_{\mathrm{f}}$ and $x_{\mathrm{sb}}$, and add integrator terms. We do not show any simulations with this standard procedure.

Instead, we investigate model disturbances in the following way. A real ASP is simulated by using our ASP model and disturbing the (output) concentrations $S_{\mathrm{f}}, u_{\mathrm{f}}, u_{\mathrm{u}}$ by multiplying these with 0.9 , i.e., the "real" outputs are $10 \%$ lower than the model outputs. In addition, we add (normal distributed) noise to these "real" signals before they enter the regulator. Since we might not know the steadystate relationship between the mass and the sludge blanket level, we assume that a reference value for the mass $m^{\text {ref }}$ (instead of $x_{\mathrm{sb}}^{\mathrm{ref}}$ ) is given in addition to $S^{\text {ref }}$.

In order to handle such disturbances, the proportional regulator in Section 6.1 is extended with integrator terms. From the conclusions in Sections 6.3 and 6.4, we set $K_{\mathrm{P}}^{S}=0 \mathrm{~m}^{3} / \mathrm{kg}$ and let the following definitions replace (36)-(37):

$$
\begin{aligned}
& r(t):=r^{\mathrm{ref}}+K_{\mathrm{I}}^{S} \int_{0}^{t}\left(S_{\mathrm{f}}(s)-S^{\mathrm{ref}}(s)\right) d s, \\
& w(t):=w^{\mathrm{ref}}+\frac{K_{\mathrm{P}}^{m}}{Q}\left(m(t)-m^{\mathrm{ref}}(t)\right)
\end{aligned}
$$



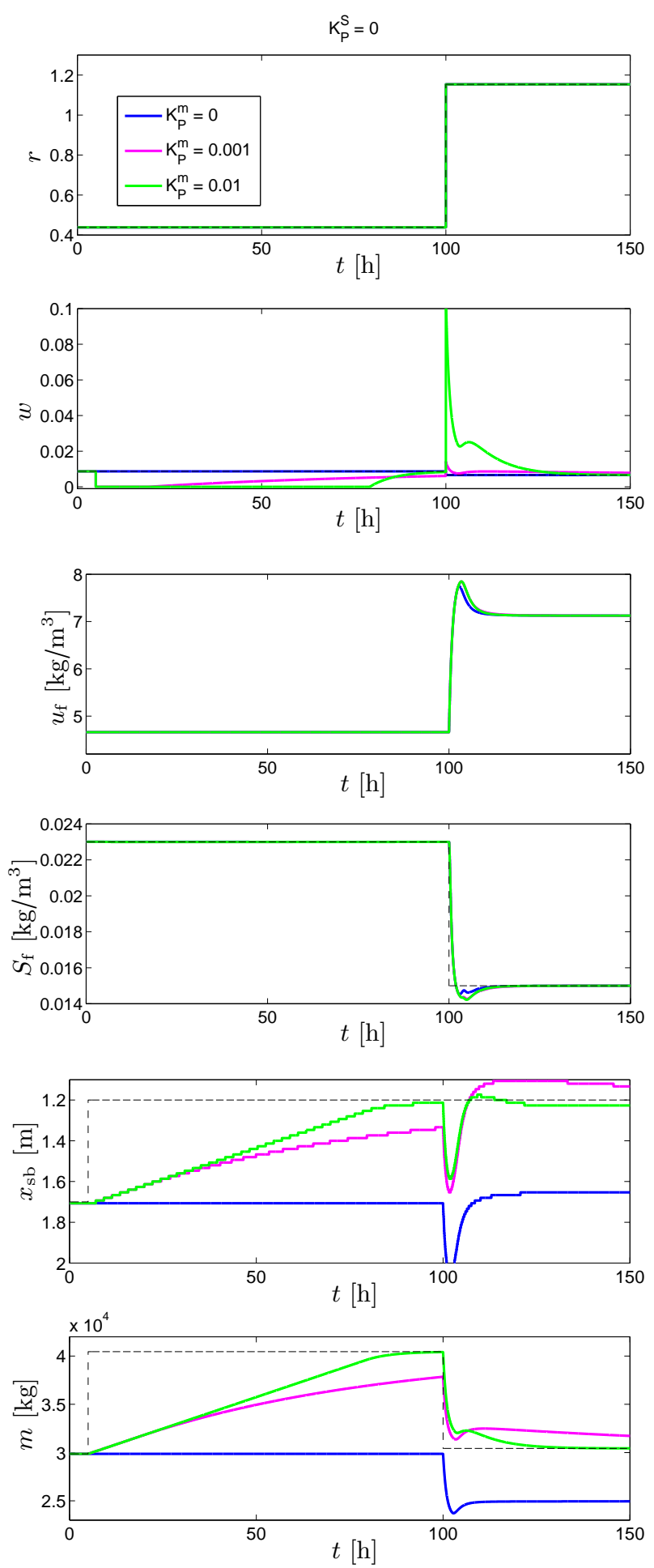

FIGURE 11. Tracking of reference values (dashed lines); step changes in $x_{\mathrm{sb}}^{\mathrm{ref}}(t)$ at $t=5 \mathrm{~h}$ and $S^{\mathrm{ref}}(t)$ at $t=100 \mathrm{~h} ; K_{\mathrm{P}}^{S}=0 \mathrm{~m}^{3} / \mathrm{kg}$ and $K_{\mathrm{P}}^{m}=0,0.001,0.01 \mathrm{~m}^{3} /(\mathrm{kgh})$. 

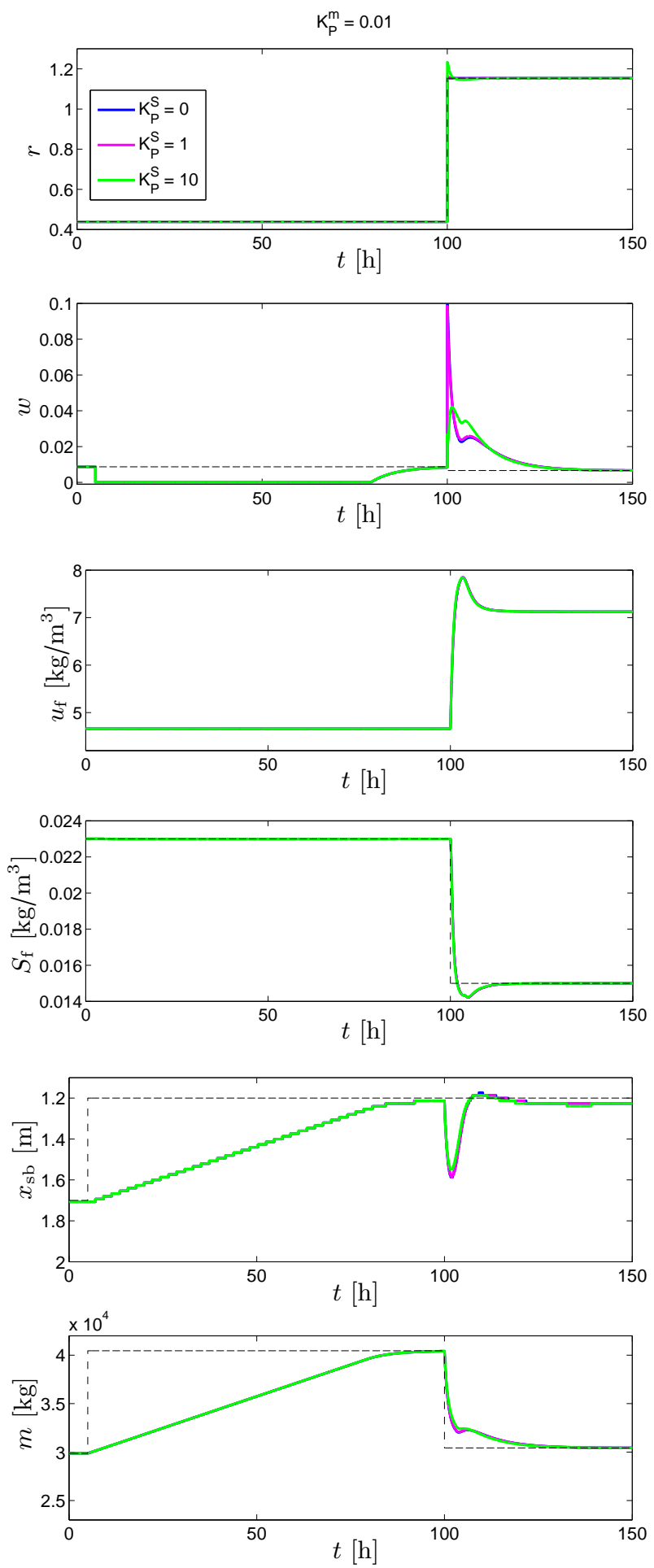

FIGURE 12. Tracking of reference values (dashed lines); step changes in $x_{\mathrm{sb}}^{\mathrm{ref}}(t)$ at $t=5 \mathrm{~h}$ and $S^{\mathrm{ref}}(t)$ at $t=100 \mathrm{~h} ; K_{\mathrm{P}}^{m}=$ $0.01 \mathrm{~m}^{3} /(\mathrm{kg} \mathrm{h})$ and $K_{\mathrm{P}}^{S}=0,1,10 \mathrm{~m}^{3} / \mathrm{kg}$. 

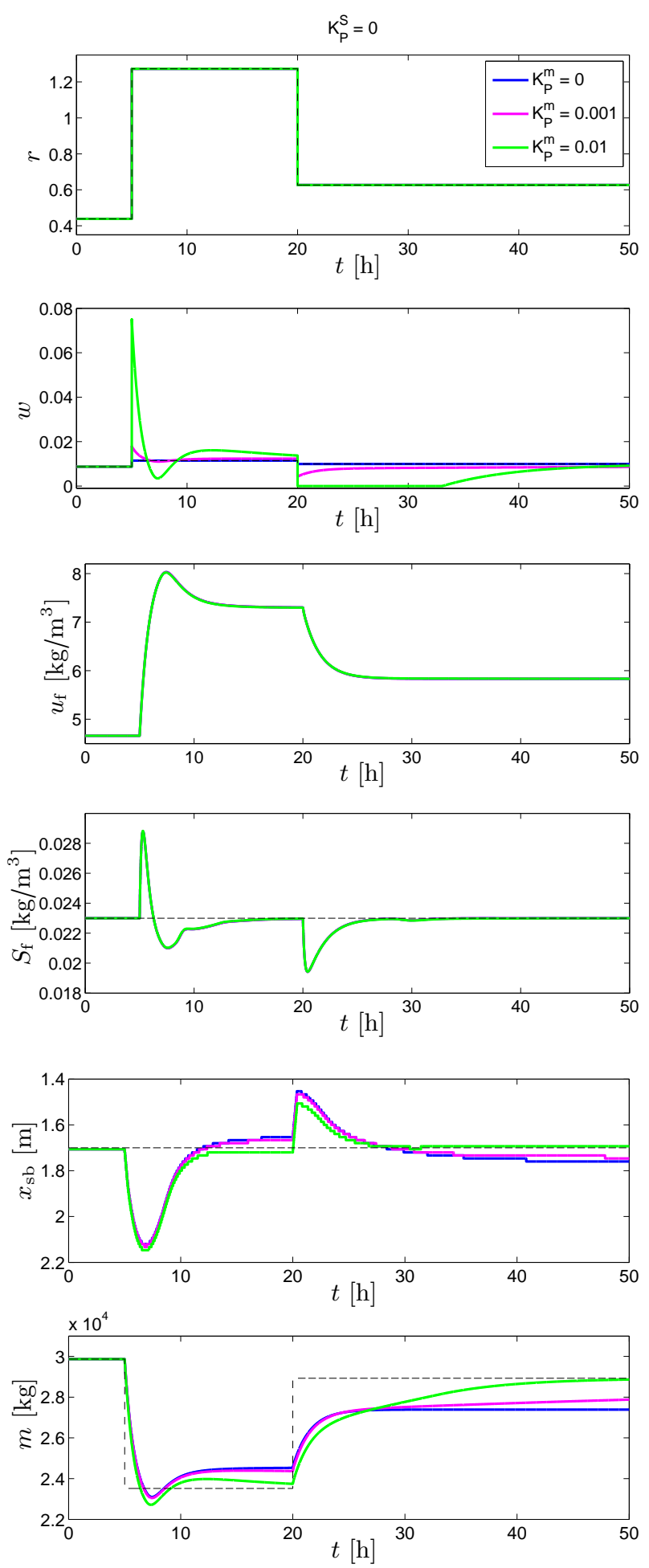

Figure 13. Closed-loop responses to load disturbances: A step increase in $S_{\text {in }}(t)$ at $t=5 \mathrm{~h}$ and a step decrease in $Q(t)$ at $t=20 \mathrm{~h}$; $K_{\mathrm{P}}^{S}=0 \mathrm{~m}^{3} / \mathrm{kg}$ and $K_{\mathrm{P}}^{m}=0,0.001,0.01 \mathrm{~m}^{3} /(\mathrm{kgh})$. 

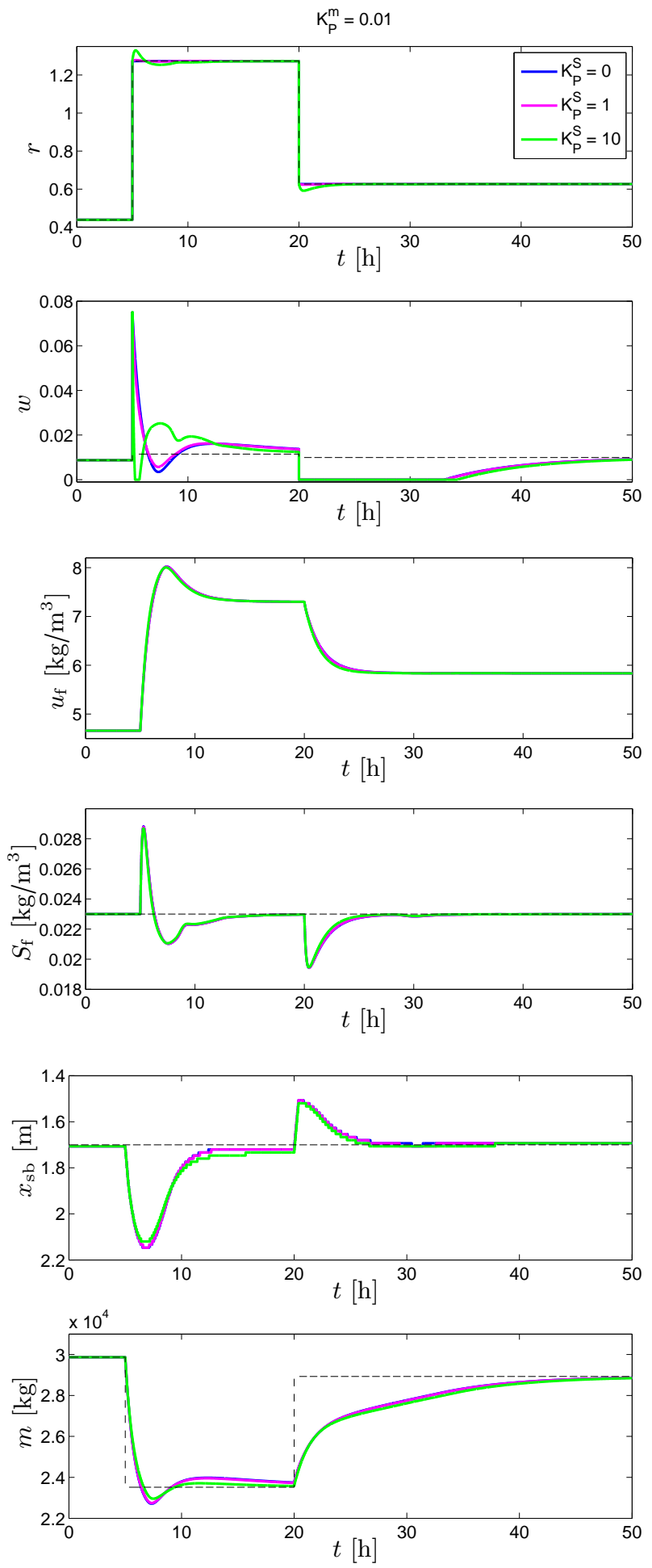

Figure 14. Closed-loop responses to load disturbances: A step increase in $S_{\text {in }}(t)$ at $t=5 \mathrm{~h}$ and a step decrease in $Q(t)$ at $t=20 \mathrm{~h}$; $K_{\mathrm{P}}^{m}=0.01 \mathrm{~m}^{3} /(\mathrm{kgh})$ and $K_{\mathrm{P}}^{S}=0,1,10 \mathrm{~m}^{3} / \mathrm{kg}$. 


$$
+\frac{K_{\mathrm{I}}^{m}}{Q} \int_{0}^{t}\left(m(s)-m^{\mathrm{ref}}(s)\right) d s-K_{\mathrm{I}}^{S} \int_{0}^{t}\left(S_{\mathrm{f}}(s)-S^{\mathrm{ref}}(s)\right) d s .
$$

A simulation with $K_{\mathrm{P}}^{m}=0.01 \mathrm{~m}^{3} /(\mathrm{kg} \mathrm{h}), K_{\mathrm{I}}^{m}=0.004 \mathrm{~m}^{3} /\left(\mathrm{kg} \mathrm{h}^{2}\right)$ and $K_{\mathrm{I}}^{S}=2 \mathrm{~m}^{3} /(\mathrm{kgh})$ is shown in Figure 15. The initial data are the same as in all previous simulations. The reference values $m^{\text {ref }}$ and $S^{\text {ref }}$ are kept constant and correspond to the previous initial normal steady-state solution. If convergence to stationary values occur, which is the case in Figure 15, the integrands in (43)-(44) have to tend to zero; hence $S_{\mathrm{f}}(t) \rightarrow S^{\text {ref }}$ and $m(t) \rightarrow m^{\text {ref }}$ as $t \rightarrow \infty$. However, the integrals in (43)-(44) may converge to non-zero values, which is clearly demonstrated by the difference between $r(t)$ and $r^{\text {ref }}$.

The advantage of coping with disturbances by the inclusion of integrator terms in (43)-(44) should be balanced by the disadvantage of integral windup which may appear when tracking reference values. As can be seen in Figures 11-12, where there are step changes in the reference values, it is in particular the slow response of $m(t)$ that will cause the mass integral windup in (44). Hence, some anti-windup measure has to be taken. From a wastewater-treatment-application point of view, there is no reason for changing the reference values $S^{\text {ref }}$ and $x_{\mathrm{sb}}^{\text {ref }}$ fast, especially not with large steps as is done in Figures 11-12. A simple anti-windup solution for this case would be to change the reference values continuously with an upper bound on their derivatives; e.g. $\left|\left(x_{\mathrm{sb}}^{\mathrm{ref}}\right)^{\prime}(t)\right| \leq C$, for some constant $C$, which can easily be estimated from Figure 11 or 12 . However, this is not sufficient for load disturbances, which may vary fast and be large in a wastewater treatment plant, and imply a fast change in $m^{\text {ref }}(t)$; see Figures $13-14$ at $t=20 \mathrm{~h}$. One solution is to invoke the integral terms only when $S_{\mathrm{f}}(t)$ and $m(t)$ lie sufficiently close to their reference values and start the integration from that time point.

\section{Conclusions}

For an ideal ASP with only two material components, modelled by an ODE-PDE system, the control objective is to keep the effluent substrate concentration at a reference value $S^{\text {ref }}$ and the concentration profile in the settler such that there is a sludge blanket at a prescribed depth $x_{\mathrm{sb}}^{\mathrm{ref}}$ in the thickening zone. This objective can be satisfied without direct measurement of $x_{\mathrm{sb}}$ or $S_{\mathrm{f}}$. The control strategy is given by an algorithm (Section 6.1), which contains a two-variable regulator for the substrate concentration and the mass in the settler.

PDE theory has been utilized to define suitable reference values $r^{\text {ref }}$ and $w^{\text {ref }}$ for the regulator. This is the key ingredient in the regulator and yields one of the main results; the control objective can be reached in steady state for constant inputs (Theorem 6.1). Another result is that the closed-loop system is locally stable with respect to load disturbances (Theorem 6.2). A main conclusion is that these results hold when a proportional regulator term for the mass is used and the feedback control of the substrate concentration is left out (i.e. $K_{\mathrm{P}}^{S}=0$ ). This is also supported by dynamic simulations; the value of $K_{\mathrm{P}}^{S}$ hardly influences the behaviour of the biological processes. Generally, the responses of the closed-loop system to different disturbances show that the biological processes are much faster than the settling process. This indicates that control of the transient behaviour of the reactor processes requires local control loops, e.g. by controlling the supply of oxygen. A conclusion is therefore that all previous publications with detailed 

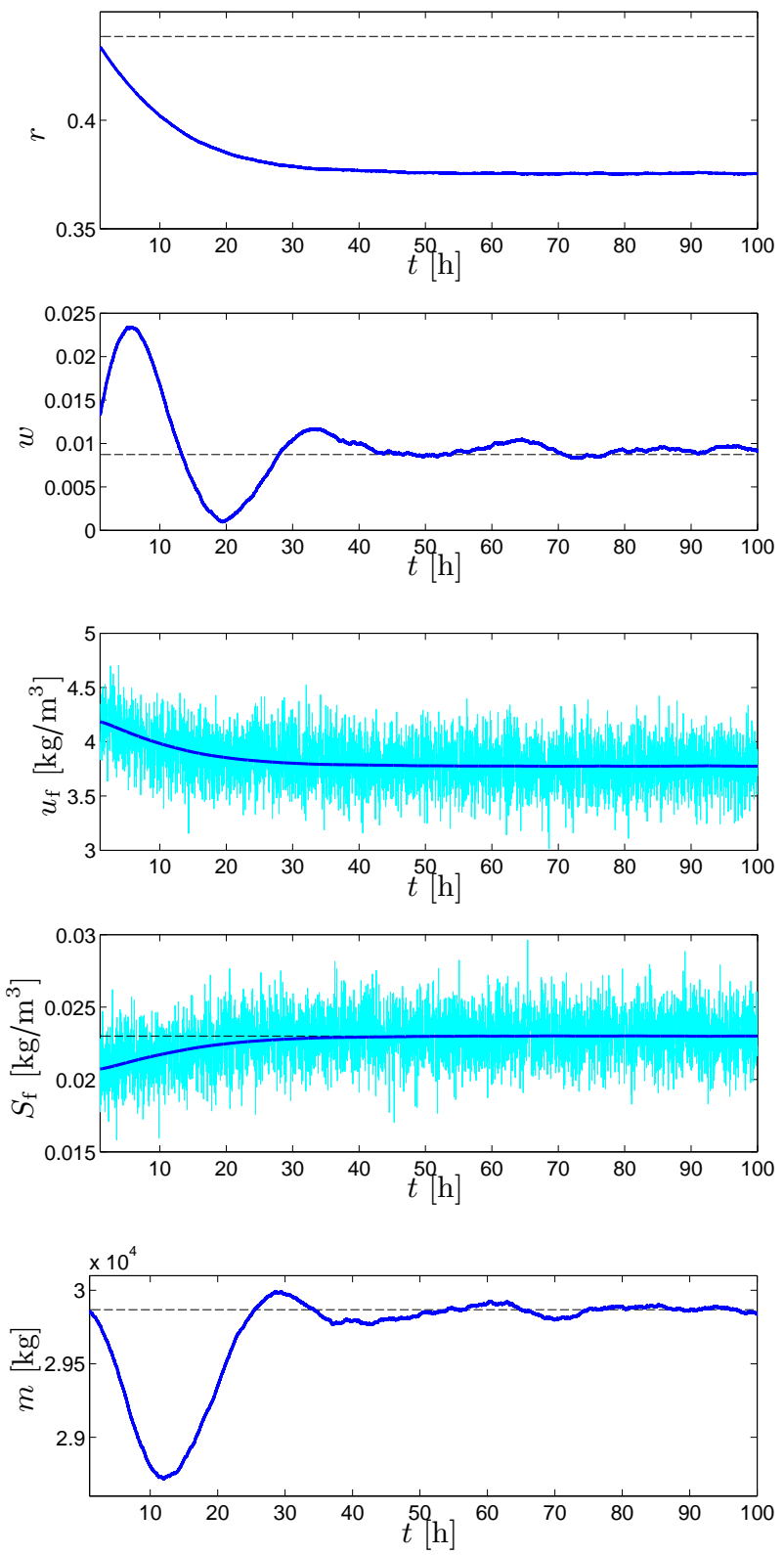

FiguRE 15. A simulation with the regulator (43)-(44) under model uncertainties of $10 \%$ (blue curves, "real" data) and noisy measurements of $u_{\mathrm{f}}$ and $S_{\mathrm{f}}$ (cyan curves), which enter the regulator. The dashed lines show the reference values.

control strategies for the biological processes (see the references in Section 1) are indeed valuable within small time scales (within some hours) despite many of them have a (too) simple settler model. The results here on the overall control of the ASP can therefore be seen as a complement to those publications. 
To cope with model uncertainties and measurement noise in the signals that enter the regulator, integrator terms for both the mass and the substrate concentration have to be added (Section 6.5). Since the large mass in the settler can only be changed slowly, integral windup has to be prevented after large load disturbances by, for example, only invoking the integrator terms when the measured signals lie sufficiently close to their reference values. As for the tunings of $K_{\mathrm{I}}^{m}$ and $K_{\mathrm{I}}^{S}$ in (44), one should start with small values and bear in mind that the control variable $w$ should normally lie around $1 \%$. In addition to our recommendation to set $K_{\mathrm{P}}^{S}=0$, a guideline for the tuning of the most important parameter in the regulator $K_{\mathrm{P}}^{m}$ is to let it be around $0.001-0.01 \mathrm{~m}^{3} /(\mathrm{kg} \mathrm{h})$ for the investigated example plant. The lower value means lower $w$ but slow convergence after step changes. The higher value is recommended under the constraint that the reference values $S^{\text {ref }}$ and $x_{\mathrm{sb}}^{\mathrm{ref}}$ should not be changed rapidly.

The reference values of the regulator originate from Theorem 4.1, which states that the desired steady-state solutions with a critically loaded settler can be parameterized by means of the main control variable $r$. This theorem also gives the conditional curve $w=W(r)$, the control curve, in the $r w$-plane; see Figures 4 (right), 6-8. For the example parameter values used, it turns out that the function values of $W$ are approximately $1 \%$ for all realistic $r$-values (see Figure 4 ). This is in agreement with real plants, despite the simplified model. An important property is that the substrate concentration for a critically loaded settler $S^{\mathrm{CL}}$ is monotone along the control curve, which implies that a point $\left(r^{\mathrm{ref}}, w^{\mathrm{ref}}\right)$ is uniquely determined for a given reference value $S^{\text {ref }}$.

The reduced-order ASP model captures the importance of recirculation. The rapidly decreasing substrate concentration $S^{\mathrm{CL}}$ along the control curve as $r$ increases from 0; see Figure 5 (left), clearly demonstrates that already a small recirculation improves the process significantly in reducing $S^{\mathrm{CL}}$. For larger $r$-values, $S^{\mathrm{CL}}$ is only slowly decreasing, and the energy cost for pumping the suspension within the plant is larger.

Challenges for the future are the inclusion of more material components; First of all, the dead particulate material should be taken into account. With a full model of the biological reactor, e.g. a system of $20 \mathrm{ODEs}$, it is a challenge to find analogous steady-state relations which can be used for the definitions of $r^{\text {ref }}$ and $w^{\text {ref }}$ in a regulator. An additional constitutive assumption for the sedimentation process, namely the compression of particles at high concentrations, is also a natural extension. This leads to a degenerate parabolic PDE for the particulate material; see Bürger et al. [7, 9].

\section{APPENDIX A. LEMMAS AND PROOFS OF THEOREMS}

In order to prove Theorem 4.1, we need the following two lemmas. They state how certain functions depend on $Q_{\mathrm{u}}=(1+r) Q$ (which is the control parameter for a stand-alone settler), $u_{\mathrm{f}}, r$ and $w$.

Lemma A.1. ([16, Appendix A])

$$
\frac{d u_{\mathrm{M}}}{d Q_{\mathrm{u}}}= \begin{cases}0, & 0 \leq Q_{\mathrm{u}}<\bar{Q}_{\mathrm{u}}, \\ -\frac{1}{A f_{\mathrm{b}}^{\prime \prime}\left(u_{\mathrm{M}}\right)}<0, & \bar{Q}_{\mathrm{u}}<Q_{\mathrm{u}}<\overline{\bar{Q}}_{\mathrm{u}}, \\ 0, & Q_{\mathrm{u}}>\overline{\bar{Q}}_{\mathrm{u}},\end{cases}
$$




$$
\begin{aligned}
& \frac{d}{d Q_{\mathrm{u}}} f\left(u_{\mathrm{M}}\left(Q_{\mathrm{u}}\right), Q_{\mathrm{u}}\right)=\frac{u_{\mathrm{M}}\left(Q_{\mathrm{u}}\right)}{A}, \quad Q_{\mathrm{u}} \geq 0, \\
& \frac{d u_{\mathrm{m}}}{d Q_{\mathrm{u}}} \stackrel{Q_{\mathrm{u}} \neq \bar{Q}_{\mathrm{u}}}{=} \frac{u_{\mathrm{M}}\left(Q_{\mathrm{u}}\right)-u_{\mathrm{m}}\left(Q_{\mathrm{u}}\right)}{A f_{\mathrm{b}}^{\prime}\left(u_{\mathrm{m}}\left(Q_{\mathrm{u}}\right)\right)+Q_{\mathrm{u}}} \begin{cases}>0, & 0 \leq Q_{\mathrm{u}}<\overline{\bar{Q}}_{\mathrm{u}}, \\
=0, & Q_{\mathrm{u}}>\overline{\bar{Q}}_{\mathrm{u}},\end{cases} \\
& \frac{\partial \Phi_{\mathrm{lim}}}{\partial Q_{\mathrm{u}}}= \begin{cases}u_{\mathrm{f}}, & u_{\mathrm{f}} \in\left(0, u_{\mathrm{m}}\left(Q_{\mathrm{u}}\right)\right) \cup\left[u_{\mathrm{M}}\left(Q_{\mathrm{u}}\right), u_{\max }\right], \\
u_{\mathrm{M}}\left(Q_{\mathrm{u}}\right), & u_{\mathrm{f}} \in\left(u_{\mathrm{m}}\left(Q_{\mathrm{u}}\right), u_{\mathrm{M}}\left(Q_{\mathrm{u}}\right)\right) .\end{cases}
\end{aligned}
$$

Lemma A.2. The following holds in $\Sigma_{r w}$ :

$$
\begin{aligned}
& \frac{\partial G}{\partial u_{\mathrm{f}}}\left(u_{\mathrm{f}}^{G}(r, w), r, w\right)=L^{\prime}\left(U_{\mathrm{f}}^{-1}\left(u_{\mathrm{f}}^{G}\right)\right) \frac{d U_{\mathrm{f}}^{-1}}{d u_{\mathrm{f}}}\left(u_{\mathrm{f}}^{G}\right)=: \gamma_{1}>0, \\
& \frac{\partial G}{\partial r}\left(u_{\mathrm{f}}^{G}(r, w), r, w\right)=-Q u_{\mathrm{f}}^{G} \frac{w(1-w)}{(r+w)^{2}}=:-\gamma_{2}<0, \\
& \frac{\partial G}{\partial w}\left(u_{\mathrm{f}}^{G}(r, w), r, w\right)=Q u_{\mathrm{f}}^{G} \frac{r(1+r)}{(r+w)^{2}}=: \gamma_{3}>0, \\
& \frac{\partial E}{\partial u_{\mathrm{f}}}\left(u_{\mathrm{f}, \lim }(r, w), r, w\right) \\
& = \begin{cases}\left.-A \frac{\partial g}{\partial u_{\mathrm{f}}}\left(u_{\mathrm{f}, \lim },(1-w) Q\right)\right)=: \varepsilon_{1 \mathrm{a}}>0, & u_{\mathrm{f}, \lim } \in\left(0, u_{\mathrm{m}}\left(Q_{\mathrm{u}}\right)\right) \cup\left[u_{\mathrm{M}}\left(Q_{\mathrm{u}}\right), u_{\max }\right), \\
\text { not defined, } & u_{\mathrm{f}, \lim }=u_{\mathrm{m}}\left(Q_{\mathrm{u}}\right), \\
(1+r) Q=: \varepsilon_{1 \mathrm{~b}}>0, & u_{\mathrm{f}, \lim } \in\left(u_{\mathrm{m}}\left(Q_{\mathrm{u}}\right), u_{\mathrm{M}}\left(Q_{\mathrm{u}}\right)\right),\end{cases} \\
& \frac{\partial E}{\partial r}\left(u_{\mathrm{f}, \lim }(r, w), r, w\right) \\
& = \begin{cases}0, & u_{\mathrm{f}, \lim } \in\left(0, u_{\mathrm{m}}\left(Q_{\mathrm{u}}\right)\right) \cup\left[u_{\mathrm{M}}\left(Q_{\mathrm{u}}\right), u_{\max }\right), \\
\text { not defined } & u_{\mathrm{f}, \lim }=u_{\mathrm{m}}\left(Q_{\mathrm{u}}\right), \\
Q\left(u_{\mathrm{f}, \mathrm{lim}}-u_{\mathrm{M}}\left(Q_{\mathrm{u}}\right)\right)=:-\varepsilon_{2 \mathrm{~b}}<0, & u_{\mathrm{f}, \lim } \in\left(u_{\mathrm{m}}\left(Q_{\mathrm{u}}\right), u_{\mathrm{M}}\left(Q_{\mathrm{u}}\right)\right),\end{cases} \\
& \frac{\partial E}{\partial w}\left(u_{\mathrm{f}, \lim }(r, w), r, w\right) \\
& = \begin{cases}-Q u_{\mathrm{f}, \lim }=:-\varepsilon_{3 \mathrm{a}}<0, & u_{\mathrm{f}, \lim } \in\left(0, u_{\mathrm{m}}\left(Q_{\mathrm{u}}\right)\right) \cup\left[u_{\mathrm{M}}\left(Q_{\mathrm{u}}\right), u_{\max }\right), \\
\text { not defined, } & u_{\mathrm{f}, \lim }=u_{\mathrm{m}}\left(Q_{\mathrm{u}}\right), \\
-Q u_{\mathrm{M}}\left(Q_{\mathrm{u}}\right)=:-\varepsilon_{3 \mathrm{~b}}<0, & u_{\mathrm{f}, \lim } \in\left(u_{\mathrm{m}}\left(Q_{\mathrm{u}}\right), u_{\mathrm{M}}\left(Q_{\mathrm{u}}\right)\right) .\end{cases}
\end{aligned}
$$

The following properties hold: $u_{\mathrm{f}}^{G}(r, \cdot)$ is decreasing; $E \in C^{1}(\Sigma \backslash I)$, where

$$
I:=\left\{\left(u_{\mathrm{f}}, r, w\right) \in \Sigma: J\left(u_{\mathrm{f}}, r, w\right):=u_{\mathrm{f}}-u_{\mathrm{m}}((r+w) Q)=0\right\} ;
$$

$E(\cdot, r, w)$ is increasing in a neighbourhood of $u_{\mathrm{f}}=u_{\mathrm{f}, \lim }(r, w) ; E\left(u_{\mathrm{f}}, r, \cdot\right)$ is decreasing; $u_{\mathrm{f}, \lim }(r, \cdot)$ is increasing; $u_{\mathrm{f}, \lim }(\cdot, r)$ is nondecreasing.

Proof. The proof of Theorem 3.1 (which can be found in [23]) gives that for the unique normal solution $S=U_{\mathrm{f}}^{-1}\left(u_{\mathrm{f}}^{G}\right), L^{\prime}(S)<0$ holds. Together with the fact that $U_{\mathrm{f}}$ is decreasing, see (15), hence $U_{\mathrm{f}}^{-1}$ is decreasing, we conclude that (46) holds. The partial derivatives of $E$ are obtained with the aim of Lemma A.1. Note that $g^{\prime}\left(u_{\mathrm{f}, \mathrm{lim}}\right)<0$ in $(47)$, because $u_{\mathrm{f}, \lim }$ is by definition the positive zero of $g\left(u_{\mathrm{f}}\right)=0$. The continuities of the partial derivatives of $E$ away from the surface $u_{\mathrm{f}}=$ $u_{\mathrm{m}}((r+w) Q)$ are easily seen, except for $\partial E / \partial u_{\mathrm{f}}$ on the surface $u_{\mathrm{f}}=u_{\mathrm{M}}((r+w) Q)$, 
where we can conclude that

$$
\varepsilon_{1 a}=-A g^{\prime}\left(u_{\mathrm{M}}\right)=-A f_{\mathrm{b}}^{\prime}\left(u_{\mathrm{M}}\right)+(1-w) Q=-A f^{\prime}\left(u_{\mathrm{M}}\right)+(1+r) Q=(1+r) Q=\varepsilon_{1 b} .
$$

Differentiation of the equation $G\left(u_{\mathrm{f}}^{G}(r, w), r, w\right)=Q\left(Y S_{\mathrm{in}}+u_{\mathrm{in}}\right)$ yields

$$
\frac{\partial u_{\mathrm{f}}^{G}}{\partial w}(r, w)=-\left.\frac{\frac{\partial G}{\partial w}}{\frac{\partial G}{\partial u_{\mathrm{f}}}}\right|_{u_{\mathrm{f}}=u_{\mathrm{f}, \lim }(r, w)}<0,
$$

which implies that $u_{\mathrm{f}}^{G}(r, \cdot)$ is decreasing. Away from the surface $I$, the monotonicity properties follow from (47)-(48) and by differentiating $\left.E\left(u_{\mathrm{f}, \lim }(r, w), r, w\right)\right)=0$, which leads to

$$
\frac{\partial u_{\mathrm{f}, \lim }}{\partial w}(r, w)=-\left.\frac{\frac{\partial E}{\partial w}}{\frac{\partial E}{\partial u_{\mathrm{f}}}}\right|_{u_{\mathrm{f}}=u_{\mathrm{f}, \lim }(r, w)}>0 .
$$

The continuous function $E(\cdot, r, w)$ has a cusp at $u_{\mathrm{f}}=u_{\mathrm{m}}$. Replace $E$ by a sequence of functions $\tilde{E}^{h} \in C^{1}$ converging towards $E$ as $h \searrow 0$ and where $\nabla \tilde{E}^{h}\left(u_{\mathrm{f}, \lim }(r, w), r, w\right)=$ $\left(\varepsilon_{1}^{h},-\varepsilon_{2}^{h},-\varepsilon_{3}^{h}\right)$ satisfies $0<\varepsilon_{1 \mathrm{a}} \leq \varepsilon_{1}^{h} \leq \varepsilon_{1 \mathrm{~b}}, 0 \leq \varepsilon_{2}^{h} \leq \varepsilon_{2 \mathrm{~b}}$ and $0<\varepsilon_{3 \mathrm{a}} \leq \varepsilon_{3}^{h} \leq \varepsilon_{3 \mathrm{~b}}$ or all $h>0$ and $(r, w) \in \Sigma_{r w}$. The equation $\tilde{E}^{h}=0$ has the solution $u_{\mathrm{f}, \lim }^{h} \in C^{1}$ (cf. [23, Lemma 3.1]), and the monotonicity properties follow from the partial derivatives above.

Lemma A.3. The surface $u_{\mathrm{f}}=u_{\mathrm{f}, \mathrm{lim}}(r, w)$ lies completely above (greater $u_{\mathrm{f}}$-values) the surface I if and only if (29) holds.

Proof. Note that $u_{\mathrm{m}}((r+w) Q)$ increases with both $r$ and $w$, and reaches its maximum value $u_{\text {inf }} \approx 5.71 \mathrm{~kg} / \mathrm{m}^{3}$ along the line $(r+w) Q=\overline{\bar{Q}}_{\mathrm{u}}$, cf. (45). For the parameter values (11), the intersecting curve in Figure 3 (right) lies always above the surface $I$ within $\Sigma$. A condition for this is the following:

$$
\begin{gathered}
u_{\mathrm{f}, \lim }(r, w)>u_{\mathrm{m}}((r+w) Q) \quad \Longleftrightarrow \quad E\left(u_{\mathrm{m}}((r+w) Q), r, w\right)<0 \quad \Longleftrightarrow \\
\left.g\left(u_{\mathrm{m}}((r+w) Q),(1-w) Q\right)\right)>0 \quad \Longleftrightarrow \quad v_{\mathrm{s}}\left(u_{\mathrm{m}}((r+w) Q)\right)>(1-w) \frac{Q}{A} .
\end{gathered}
$$

Set $R(r, w):=v_{\mathrm{s}}\left(u_{\mathrm{m}}((r+w) Q)\right)-(1-w) Q / A$ for $(r, w) \in \Sigma_{r w}$. Then $R(\cdot, w)$ is decreasing since $u_{\mathrm{m}}$ is increasing by Lemma A.1 and $v_{\mathrm{s}}$ is decreasing by assumption. Along the boundary of $\Sigma_{r w}$ with the equation $(r+w) Q=\overline{\bar{Q}}_{\mathrm{u}}$, we have $R\left(\overline{\bar{Q}}_{\mathrm{u}} / Q-\right.$ $w, w)=v_{\mathrm{s}}\left(u_{\mathrm{infl}}\right)-(1-w) Q / A$, which is a decreasing function of $w$. In particular, $w=$ 1 yields the value $R\left(\overline{\bar{Q}}_{\mathrm{u}} / Q-1,1\right)=v_{\mathrm{s}}\left(u_{\mathrm{infl}}\right)>0$. The minimum value is $R\left(\overline{\bar{Q}}_{\mathrm{u}} / Q-\right.$ $0,0)=v_{\mathrm{s}}\left(u_{\mathrm{infl}}\right)-Q / A$. Hence, if $(29)$ holds, then the surface $u_{\mathrm{f}}=u_{\mathrm{f}, \lim }(r, w)$ lies completely above $I$. Otherwise, $R(r, w)=0$ defines a curve in $\Sigma_{r w}$ that cuts the line $(r+w) Q=\overline{\bar{Q}}_{\mathrm{u}}$.

Proof. (Theorem 4.1) Step 1, prerequisites: The prerequisite inequality (16) is the same as in Theorem 3.1. Since $0 \leq w<1$, (26) implies $(17)$ for $(r, w) \in \Sigma_{r w}$. If $u_{\text {in }}>0$, then according to Theorem 3.1, there exists a unique normal solution $u_{\mathrm{f}}^{G}(r, w) \in\left(0, u_{\max }\right]$ for every $(r, w) \in \Sigma_{r w}$. If $u_{\text {in }}=0$, then the existence of a nonzero $u_{\mathrm{f}}^{G}(r, w) \in\left(0, u_{\max }\right]$ is only guaranteed for a subset of $\Sigma_{r w}$, namely those $(r, w)$ satisfying (17). To investigate this, we substitute $S_{\lim }(r, w)$, which is the solution $S$ of $E\left(U_{\mathrm{f}}^{-1}(S), r, w\right)=0$, into $(24)$ and rewrite the equation by means of (12):

$$
G\left(U_{\mathrm{f}}\left(S_{\lim }(r, w)\right), r, w\right)=Y Q S_{\mathrm{in}} \quad \Longleftrightarrow
$$




$$
\begin{gathered}
U_{\mathrm{f}}\left(S_{\lim }\right)(\alpha(r, w)+b \tau)+Y S_{\lim }=Y S_{\mathrm{in}} \quad \Longleftrightarrow \\
\alpha(r, w)=\left(\mu\left(S_{\lim }\right)-b\right) \tau .
\end{gathered}
$$

Since $\alpha(r, w) \in(0,1)$ for all $(r, w) \in \Sigma_{r w}$, (50) implies that (17) is clearly true if $\left(\mu\left(S_{\mathrm{in}}\right)-b\right) \tau \geq 1$ and false if $\left(\mu\left(S_{\mathrm{in}}\right)-b\right) \tau \leq 0$. The latter proves case $b$. In the intermediate case $0<\left(\mu\left(S_{\text {in }}\right)-b\right) \tau<1$, we use that $\mu$ is increasing to establish that (17) holds:

$$
\alpha(r, w)=\left(\mu\left(S_{\lim }\right)-b\right) \tau<\left(\mu\left(S_{\text {in }}\right)-b\right) \tau .
$$

Hence, for $u_{\text {in }}=0,(17)$ is satisfied if and only if $\mu\left(S_{\text {in }}\right)>b$. Then according to Theorem 3.1 there exists a unique normal solution $u_{\mathrm{f}}^{G}(r, w) \in\left(0, u_{\max }\right]$ for every $(r, w) \in \Sigma_{r w}$. Step 2, uniqueness: By (46) and the implicit function theorem, $u_{\mathrm{f}}^{G} \in C^{1}$ holds. The monotonicity properties of the Lipschitz continuous function $E(\cdot, r, w)$ in Lemma A.2, together with the corresponding version of the implicit function theorem, implies that $u_{\mathrm{f}, \mathrm{lim}}(\mathrm{see}(23)$ ) is a continuous function. Away from the surface $I$, see $(49), u_{\mathrm{f}, \mathrm{lim}} \in C^{1}$ holds. Lemma A.2 also states that, for every fixed $r_{0}>0, u_{\mathrm{f}, \lim }\left(r_{0}, \cdot\right)$ is increasing and $u_{\mathrm{f}}^{G}\left(r_{0}, \cdot\right)$ is decreasing. Hence, if, for given $r_{0}>0$, there exists a $w_{0} \in[0,1)$ satisfying $u_{\mathrm{f}, \lim }\left(r_{0}, w_{0}\right)=u_{\mathrm{f}}^{G}\left(r_{0}, w_{0}\right)$, then it is unique and we can define a function $W$ via $W\left(r_{0}\right):=w_{0}$. Step 3, existence: We shall now prove the existence of an intersection of the two surfaces $u_{\mathrm{f}}=u_{\mathrm{f}, \lim }(r, w)$ and $u_{\mathrm{f}}=u_{\mathrm{f}}^{G}(r, w)$ for small $r_{0}$. Start with $r_{0}=0$ on the boundary of $\Sigma_{r w}$. Note that $u_{\mathrm{f}}^{G}(0, w)$ is independent of $w$, since it is the solution of the equation $G\left(u_{\mathrm{f}}, 0, w\right)=Q\left(Y S_{\text {in }}+u_{\text {in }}\right)$, which is equivalent to

$$
u_{\mathrm{f}}(1+b \tau)+Y U_{\mathrm{f}}^{-1}\left(u_{\mathrm{f}}\right)=Y S_{\mathrm{in}}+u_{\mathrm{in}} .
$$

As in the proof of Theorem 3.1, one can conclude that the constant $\xi_{0}:=u_{\mathrm{f}}^{G}(0, w) \epsilon$ $\left(0, u_{\max }\right]$ for every $w \in(0,1)$. We also note that $u_{\mathrm{f}}^{G}(r, 1)=\xi_{0}$, since $G\left(u_{\mathrm{f}}, r, 1\right)=$ $G\left(u_{\mathrm{f}}, 0, w\right)$ is independent of both $r$ and $w$. Since $u_{\mathrm{f}}^{G}(r, \cdot)$ is decreasing and takes values in $\left(0, u_{\max }\right]$, we conclude that $0<\xi_{0}<u_{\max }$ must hold. Thus, $u_{\mathrm{f}}^{G}\left(r_{0}, w\right)<$ $u_{\mathrm{f}}^{G}\left(r_{0}, 0\right)$ holds, where $u_{\mathrm{f}}^{G}\left(r_{0}, 0\right)$ actually satisfies (51). Since this equation is independent of $r_{0}, u_{\mathrm{f}}^{G}\left(r_{0}, w\right)<u_{\mathrm{f}}^{G}\left(r_{0}, 0\right)=$ constant for all $r_{0}>0$ and $w \in(0,1]$. This implies the equality of $(28)$. The increasing and continuous function $u_{\mathrm{f}, \lim }(0, \cdot)$ satisfies $u_{\mathrm{f}, \lim }(0,0)=0$, since

$$
0=E\left(u_{\mathrm{f}, \lim }(0,0), 0,0\right)=Q u_{\mathrm{f}, \lim }(0,0)-\Phi_{\lim }\left(u_{\mathrm{f}, \lim }(0,0), 0\right)=Q u_{\mathrm{f}, \lim }(0,0) .
$$

It also satisfies

$$
\begin{aligned}
& E\left(u_{\mathrm{f}, \lim }(r, 1), r, 1\right) \\
= & \begin{cases}-A f_{\mathrm{b}}\left(u_{\mathrm{f}, \lim }(r, 1)\right), & u_{\mathrm{f}, \lim }(r, 1) \in\left[0, u_{\mathrm{m}}\right] \cup\left[u_{\mathrm{M}}, u_{\mathrm{max}}\right], \\
(1+r) Q\left(u_{\mathrm{f}, \lim }(r, 1)-u_{\mathrm{M}}\right)-A f_{\mathrm{b}}\left(u_{\mathrm{M}}\right)<0, & u_{\mathrm{f}, \lim }(r, 1) \in\left(u_{\mathrm{m}}, u_{\mathrm{M}}\right),\end{cases}
\end{aligned}
$$

which means that

$$
E\left(u_{\mathrm{f}, \lim }(r, 1), r, 1\right)=0 \quad \Longleftrightarrow \quad f_{\mathrm{b}}\left(u_{\mathrm{f}, \lim }(r, 1)\right)=0 \quad \Longleftrightarrow \quad u_{\mathrm{f}, \lim }(r, 1)=u_{\max }
$$

for all $r \geq 0$. In particular, $u_{\mathrm{f}, \lim }(0, \cdot)$ increases from $u_{\mathrm{f}, \lim }(0,0)=0$ to $u_{\mathrm{f}, \lim }(0,1)=$ $u_{\max }$. Hence, there exists a unique $w_{0} \in(0,1)$ such that $u_{\mathrm{f}, \lim }\left(0, w_{0}\right)=\xi_{0}=$ $u_{\mathrm{f}}^{G}\left(0, w_{0}\right)$. Therefore, the point $\boldsymbol{\sigma}(0)=\left(u_{\mathrm{f}}^{\mathrm{CL}}(0), 0, W(0)\right):=\left(\xi_{0}, 0, w_{0}\right)$ satisfies both Eqs (23) and (24). Starting in this point, the intersection of (23) and (24) defines a continuous curve (by the monotonicity properties of Lemma A.2) for increasing $r$ as long as $(28)$ holds, since $u_{\mathrm{f}, \mathrm{lim}}(\cdot, 0)$ is increasing by Lemma A.2. The 
monotonicity properties of $u_{\mathrm{f}, \lim }$ and $u_{\mathrm{f}}^{G}$ yield that $W\left(r_{1}\right)=0$ for $r_{1}$ equal to the infimum of the $r$ for which (28) is violated. Step 4, regularity and monotonicity: Lemma A.2 implies that

$$
\begin{aligned}
& \nabla G \times \nabla E(\boldsymbol{\sigma}(r)) \\
& = \begin{cases}\left(\varepsilon_{3 \mathrm{a}} \gamma_{2}, \varepsilon_{1 \mathrm{a}} \gamma_{3}+\varepsilon_{3 \mathrm{a}} \gamma_{1}, \varepsilon_{1 \mathrm{a}} \gamma_{2}\right), & u_{\mathrm{f}}^{\mathrm{CL}}(r) \in\left(0, u_{\mathrm{m}}\right] \cup\left[u_{\mathrm{M}}, u_{\mathrm{max}}\right], \\
\text { not defined, } & u_{\mathrm{f}}^{\mathrm{CL}}(r)=u_{\mathrm{m}}, \\
\left(\varepsilon_{3 \mathrm{~b}} \gamma_{2}+\varepsilon_{2 \mathrm{~b}} \gamma_{3}, \varepsilon_{1 \mathrm{~b}} \gamma_{3}+\varepsilon_{3 \mathrm{~b}} \gamma_{1}, \varepsilon_{1 \mathrm{~b}} \gamma_{2}-\varepsilon_{2 \mathrm{~b}} \gamma_{1}\right), & u_{\mathrm{f}}^{\mathrm{CL}}(r) \in\left(u_{\mathrm{m}}, u_{\mathrm{M}}\right) .\end{cases}
\end{aligned}
$$

Hence, for every $r \in\left[0, \overline{\bar{Q}}_{\mathrm{u}} / Q\right]$ for which $u_{\mathrm{f}}^{\mathrm{CL}}(r) \neq u_{\mathrm{m}}((r+W(r)) Q)$ holds, the second component of the vector $\nabla G \times \nabla E(\sigma(r))$ is positive. The implicit function theorem then implies that $\boldsymbol{\sigma} \in C^{1}$ with $\boldsymbol{\sigma}^{\prime}(r) \| \nabla G \times \nabla E(\boldsymbol{\sigma}(r))$. The first component of the latter parallel vectors is also positive so that $\left(u_{\mathrm{f}}^{\mathrm{CL}}\right)^{\prime}(r)>0$ for $r>0$ away from $I$. The continuous function $E(\cdot, r, w)$ has a cusp at $u_{\mathrm{f}}=u_{\mathrm{m}}$. As in the proof of Lemma A.2 we can replace $E$ by a sequence of functions $\tilde{E}_{h} \in C^{1}$ converging to $E$ as $h \searrow 0$ and where $\nabla \tilde{E}^{h}\left(u_{\mathrm{f}, \lim }(r, w), r, w\right)=\left(\varepsilon_{1}^{h},-\varepsilon_{2}^{h},-\varepsilon_{3}^{h}\right)$ satisfies $0<\varepsilon_{1 \mathrm{a}} \leq \varepsilon_{1}^{h} \leq \varepsilon_{1 \mathrm{~b}}$ and $0<\varepsilon_{3 \mathrm{a}} \leq \varepsilon_{3}^{h} \leq \varepsilon_{3 \mathrm{~b}}$ or all $h>0$. Computing $\nabla G \times \nabla \tilde{E}_{h}(\boldsymbol{\sigma}(r))$, we can draw the same conclusions as above for every fixed $h>0$. Since $\varepsilon_{1}^{h}$ and $\varepsilon_{3}^{h}$ are bounded away from zero, we can conclude that $u_{\mathrm{f}}^{\mathrm{CL}}$ is indeed increasing.

Proof. (Theorem 5.1) The governing Eqs (23), (24) and $u_{\mathrm{f}}=U_{\mathrm{f}}(S)$ can be written

$$
\begin{aligned}
& \left\{\begin{array}{l}
G\left(u_{\mathrm{f}}^{\mathrm{CL}}\left(r, S_{\text {in }}, u_{\text {in }}\right), r, W\left(r, S_{\text {in }}, u_{\text {in }}\right)\right)=Q\left(Y S_{\text {in }}+u_{\text {in }}\right) \\
E\left(u_{\mathrm{f}}^{\mathrm{CL}}\left(r, S_{\text {in }}, u_{\text {in }}\right), r, W\left(r, S_{\text {in }}, u_{\text {in }}\right)\right)=0 \\
u_{\mathrm{f}}^{\mathrm{CL}}\left(r, S_{\text {in }}, u_{\text {in }}\right)=U_{\mathrm{f}}\left(S^{\mathrm{CL}}\left(r, S_{\text {in }}, u_{\text {in }}\right), S_{\text {in }}\right)
\end{array} \Longleftrightarrow\right. \\
& \left\{\begin{array}{l}
u_{\mathrm{f}}^{\mathrm{CL}}\left(r, S_{\mathrm{in}}, u_{\text {in }}\right)\left(\alpha\left(r, W\left(r, S_{\mathrm{in}}, u_{\text {in }}\right)\right)+b \tau\right)+Y S^{\mathrm{CL}}\left(r, S_{\mathrm{in}}, u_{\text {in }}\right)=Y S_{\text {in }}+u_{\text {in }} \\
E\left(u_{\mathrm{f}}^{\mathrm{CL}}\left(r, S_{\mathrm{in}}, u_{\text {in }}\right), r, W\left(r, S_{\text {in }}, u_{\text {in }}\right)\right)=0 \\
u_{\mathrm{f}}^{\mathrm{CL}}\left(r, S_{\mathrm{in}}, u_{\text {in }}\right) \mu\left(S^{\mathrm{CL}}\left(r, S_{\mathrm{in}}, u_{\text {in }}\right)\right) \tau=Y\left(S_{\mathrm{in}}-S^{\mathrm{CL}}\left(r, S_{\text {in }}, u_{\text {in }}\right)\right) .
\end{array}\right.
\end{aligned}
$$

We refer to the notation in Lemma A.2. By doing the same smooth regularization of $E(\cdot, r, w)$ in the vicinity of its cusp at $u_{\mathrm{f}}=u_{\mathrm{m}}$ made in the proofs of Lemma A.2 and Theorem 4.1, we can write $\varepsilon_{1}>0$ instead of $\frac{\partial E}{\partial u_{\mathrm{f}}}$ and $-\varepsilon_{3}<0$ instead of $\frac{\partial E}{\partial w}$. For simplicity, we keep the notation $E$ also for its smooth approximation. Differentiation of the three equations in (53) with respect to $S_{\text {in }}$ yields:

$$
\left(\begin{array}{ccc}
\alpha+b \tau & u_{\mathrm{f}}^{\mathrm{CL}} \alpha_{w} & Y \\
\varepsilon_{1} & -\varepsilon_{3} & 0 \\
\mu\left(S^{\mathrm{CL}}\right) \tau & 0 & u_{\mathrm{f}}^{\mathrm{CL}} \mu^{\prime}\left(S^{\mathrm{CL}}\right) \tau+Y
\end{array}\right)\left(\begin{array}{c}
\frac{\partial u_{\mathrm{f}}^{\mathrm{CL}}}{\partial S_{\text {in }}} \\
\frac{\partial W}{\partial S_{\text {in }}} \\
\frac{\partial S^{\mathrm{CL}}}{\partial S_{\text {in }}}
\end{array}\right)=\left(\begin{array}{c}
Y \\
0 \\
Y
\end{array}\right)
$$

where $\alpha_{w}:=\partial \alpha / \partial w=r(1+r) /(r+w)^{2}>0$. The determinant of the system matrix in $(54)$ is

$$
\begin{aligned}
\Gamma_{S_{\mathrm{in}}}=-\varepsilon_{1} u_{\mathrm{f}}^{\mathrm{CL}} \alpha_{w}\left(u_{\mathrm{f}}^{\mathrm{CL}} \mu^{\prime}\left(S^{\mathrm{CL}}\right) \tau+Y\right)-\varepsilon_{3}(\alpha+b \tau) u_{\mathrm{f}}^{\mathrm{CL}} \mu^{\prime}\left(S^{\mathrm{CL}}\right) \tau & \\
& -\varepsilon_{3} Y\left(\alpha+b \tau-\mu\left(S^{\mathrm{CL}}\right) \tau\right) .
\end{aligned}
$$


The first two terms are clearly negative. The last term is also negative, which can be seen by eliminating $u_{\mathrm{f}}^{\mathrm{CL}}$ from the first and third equation of (53):

$$
\frac{\alpha+b \tau}{\mu\left(S^{\mathrm{CL}}\right) \tau}=\frac{Y\left(S_{\mathrm{in}}-S^{\mathrm{CL}}\right)+u_{\text {in }}}{Y\left(S_{\mathrm{in}}-S^{\mathrm{CL}}\right)} \geq 1
$$

Hence, $\Gamma_{S_{\text {in }}}<0$ holds and the solution of $(54)$ is

$$
\begin{aligned}
& \frac{\partial u_{\mathrm{f}}^{\mathrm{CL}}}{\partial S_{\mathrm{in}}}=-\frac{\varepsilon_{3} Y u_{\mathrm{f}}^{\mathrm{CL}} \mu^{\prime}\left(S^{\mathrm{CL}}\right) \tau}{\Gamma_{S_{\mathrm{in}}}}>0, \quad \frac{\partial W}{\partial S_{\mathrm{in}}}=-\frac{\varepsilon_{1} Y u_{\mathrm{f}}^{\mathrm{CL}} \mu^{\prime}\left(S^{\mathrm{CL}}\right) \tau}{\Gamma_{S_{\mathrm{in}}}}>0, \\
& \frac{\partial S^{\mathrm{CL}}}{\partial S_{\mathrm{in}}}=-Y \frac{\varepsilon_{1} u_{\mathrm{f}}^{\mathrm{CL}} \alpha_{w}+\varepsilon_{3}\left(\alpha+b \tau-\mu\left(S^{\mathrm{CL}}\right) \tau\right)}{\Gamma_{S_{\mathrm{in}}}}>0,
\end{aligned}
$$

where we again have used (55). Differentiation of (52) with respect to $u_{\text {in }}$ yields:

$$
\left(\begin{array}{ccc}
\gamma_{1} & \gamma_{3} & 0 \\
\varepsilon_{1} & -\varepsilon_{3} & 0 \\
1 & 0 & -U_{\mathrm{f}}^{\prime}\left(S^{\mathrm{CL}}\right)
\end{array}\right)\left(\begin{array}{c}
\frac{\partial u_{\mathrm{f}}^{\mathrm{CL}}}{\partial u_{\mathrm{in}}} \\
\frac{\partial W}{\partial u_{\mathrm{in}}} \\
\frac{\partial S^{\mathrm{CL}}}{\partial u_{\mathrm{in}}}
\end{array}\right)=\left(\begin{array}{c}
Q Y \\
0 \\
0
\end{array}\right)
$$

The system determinant is $\Gamma_{u_{\text {in }}}=U_{\mathrm{f}}^{\prime}\left(S^{\mathrm{CL}}\right)\left(\gamma_{1} \varepsilon_{3}+\gamma_{3} \varepsilon_{1}\right)<0\left(\right.$ since $\left.U_{\mathrm{f}}^{\prime}\left(S^{\mathrm{CL}}\right)<0\right)$, and the derivatives are

$$
\frac{\partial u_{\mathrm{f}}^{\mathrm{CL}}}{\partial u_{\mathrm{in}}}=\frac{\varepsilon_{3} Q Y U_{\mathrm{f}}^{\prime}\left(S^{\mathrm{CL}}\right)}{\Gamma_{u_{\mathrm{in}}}}>0, \quad \frac{\partial W}{\partial u_{\mathrm{in}}}=\frac{\varepsilon_{1} Y U_{\mathrm{f}}^{\prime}\left(S^{\mathrm{CL}}\right)}{\Gamma_{u_{\mathrm{in}}}}>0, \quad \frac{\partial S^{\mathrm{CL}}}{\partial u_{\mathrm{in}}}=\frac{\varepsilon_{3} Q Y}{\Gamma_{u_{\mathrm{in}}}}<0 .
$$

Proof. (Theorem 6.2) Assume that, at $t=0$, the ASP is in steady state, the settler is in optimal operation and that there are small disturbances in the state variables $\left(S_{\mathrm{f}}, u_{\mathrm{f}}, m\right)$ of $(42)$. The qualitative behaviour is then governed by the Jacobian of (42), where $r$ and $w$ are defined by (36)-(37), and $S_{\mathrm{u}}$ or $u_{\mathrm{u}}$ are outputs from the PDEs (4)-(5). The regulator (36)-(37) yield the following:

$$
\begin{array}{lll}
\frac{\partial r}{\partial S_{\mathrm{f}}}=K_{\mathrm{P}}^{S}, & \frac{\partial r}{\partial u_{\mathrm{f}}}=0, & \frac{\partial r}{\partial m}=0, \\
\frac{\partial(r+w)}{\partial S_{\mathrm{f}}}=0, & \frac{\partial(r+w)}{\partial u_{\mathrm{f}}}=0, & \frac{\partial(r+w)}{\partial m}=\frac{K_{\mathrm{P}}^{m}}{Q} .
\end{array}
$$

The PDE output concentrations $S_{\mathrm{u}}$ or $u_{\mathrm{u}}$ are independent of the feed variables $S_{\mathrm{f}}$ and $u_{\mathrm{f}}$ for small times. This follows from the PDE theory; see $[17,19]$. A small disturbance in the state variable $m$ may, however, cause a change in $u_{\mathrm{u}}$ either directly via the mass $m(0)=A \int_{0}^{B} u(x, 0) d x$ or indirectly via the regulator. Consider first a disturbance in the concentration profile $u(x, 0)$ in the thickening zone. The bottom concentration $u\left(B^{-}, 0\right)$ may take any value in $\left[0, u_{\max }\right]$. Then the dynamic solution satisfies the following for small $t>0$ (see [14, Section 7.2]):

$$
u\left(B^{-}, t\right) \begin{cases}<u_{\mathrm{m}}, & \text { then } u_{\mathrm{u}}(t)<u_{\mathrm{u}}(0), \\ \in\left[u_{\mathrm{m}}, u_{\mathrm{M}}\right], & \text { then } u_{\mathrm{u}}(t)=u_{\mathrm{u}}(0), \\ >u_{\mathrm{M}}, & \text { then } u_{\mathrm{u}}(t)>u_{\mathrm{u}}(0) .\end{cases}
$$

Since we consider only small disturbances of $u\left(B^{-}, t\right)$ from $u\left(B^{-}, 0\right)=u_{\mathrm{M}}\left(Q_{\mathrm{u}}(0)\right)$, we get $\partial u_{\mathrm{u}} / \partial m=0$ if $u\left(B^{-}, t\right)<u_{\mathrm{M}}\left(Q_{\mathrm{u}}(0)\right)$, and $\partial u_{\mathrm{u}} / \partial m>0$ if $u\left(B^{-}, t\right)>$ $u_{\mathrm{M}}\left(Q_{\mathrm{u}}(0)\right)$. Hence, we conclude that $\partial u_{\mathrm{u}} / \partial m \geq 0$ holds. The influence of a disturbance in $m$ via the regulator occurs since $(r+w)$ changes. Consider a small change 
in the underflow rate from $Q_{\mathrm{u}}$ to $Q_{\mathrm{u}}+\Delta Q_{\mathrm{u}}$. Assume first that $\Delta Q_{\mathrm{u}}>0$. Then the dynamic behaviour for small times is given by the solution shown in [20, Figure 5], from which the following change in $u_{\mathrm{u}}$ can be derived (the bottom concentration is $u_{\mathrm{M}}\left(Q_{\mathrm{u}}\right)$ for small times):

$$
\Delta u_{\mathrm{u}}=\frac{A f\left(u_{\mathrm{M}}\left(Q_{\mathrm{u}}\right), Q_{\mathrm{u}}+\Delta Q_{\mathrm{u}}\right)}{Q_{\mathrm{u}}+\Delta Q_{\mathrm{u}}}-\frac{A f\left(u_{\mathrm{M}}\left(Q_{\mathrm{u}}\right), Q_{\mathrm{u}}\right)}{Q_{\mathrm{u}}}=-\frac{A f_{\mathrm{b}}\left(u_{\mathrm{M}}\left(Q_{\mathrm{u}}\right)\right) \Delta Q_{\mathrm{u}}}{\left(Q_{\mathrm{u}}+\Delta Q_{\mathrm{u}}\right) Q_{\mathrm{u}}} .
$$

Dividing by $\Delta Q_{\mathrm{u}}$ and letting $\Delta Q_{\mathrm{u}} \rightarrow 0$, we get

$$
\frac{\partial u_{\mathrm{u}}}{\partial Q_{\mathrm{u}}}=-\frac{A f_{\mathrm{b}}\left(u_{\mathrm{M}}\left(Q_{\mathrm{u}}\right)\right)}{Q_{\mathrm{u}}^{2}}
$$

hence,

$$
\frac{\partial u_{\mathrm{u}}}{\partial(r+w)}=Q \frac{\partial u_{\mathrm{u}}}{\partial Q_{\mathrm{u}}}=-\frac{A f_{\mathrm{b}}\left(u_{\mathrm{M}}\left(Q_{\mathrm{u}}\right)\right)}{Q(r+w)^{2}} .
$$

If $\Delta Q_{\mathrm{u}}<0$, then there will be a small expansion wave at the bottom with the new bottom value $u_{\mathrm{M}}\left(Q_{\mathrm{u}}+\Delta Q_{\mathrm{u}}\right)$; see [20, Figure 7]. A similar derivation as above gives again (56). The influence on $S_{\mathrm{u}}$ is in principle the same; however there is no batch settling flux in the PDE for $S$, which implies that $\partial S_{\mathrm{u}} / \partial(r+w)=0$. Now we get the following elements of the Jacobian $J=\left(J_{i j}\right)$ for System (42) in a normal steady state of the ASP with the settler in optimal operation (where $S_{\mathrm{u}}=S_{\mathrm{f}}$ ):

$$
\begin{aligned}
J_{11} & =K_{\mathrm{P}}^{S} S_{\mathrm{u}}-K_{\mathrm{P}}^{S} S_{\mathrm{f}}-(1+r)-\tau \frac{\mu^{\prime}\left(S_{\mathrm{f}}\right)}{Y} u_{\mathrm{f}}=-(1+r)-\tau \frac{\mu^{\prime}\left(S_{\mathrm{f}}\right)}{Y} u_{\mathrm{f}}<0, \\
J_{12} & =-\tau \frac{\mu\left(S_{\mathrm{f}}\right)}{Y}<0, \quad J_{13}=0, \\
J_{21} & =K_{\mathrm{P}}^{S} u_{\mathrm{u}}-K_{\mathrm{P}}^{S} u_{\mathrm{f}}+\tau \mu^{\prime}\left(S_{\mathrm{f}}\right) u_{\mathrm{f}}>0, \quad J_{22}=-(1+r)+\tau\left(\mu\left(S_{\mathrm{f}}\right)-b\right), \quad J_{23}=0, \\
J_{33} & =-V \frac{K_{\mathrm{P}}^{m}}{Q} u_{\mathrm{u}}-V(r+w)\left[\frac{\partial u_{\mathrm{u}}}{\partial m}+\frac{\partial u_{\mathrm{u}}}{\partial(r+w)} \frac{\partial(r+w)}{\partial m}\right] \\
& =-V \frac{K_{\mathrm{P}}^{m}}{Q} u_{\mathrm{M}}-V(r+w) \frac{\partial u_{\mathrm{u}}}{\partial m}<0,
\end{aligned}
$$

where we in the last equality have used (56). Utilizing that the right-hand side of the middle equation of (42) is zero in steady state, we note that $J_{22}=-\left(u_{\mathrm{in}}+r u_{\mathrm{u}}\right) / u_{\mathrm{f}}<0$. The eigenvalues of $J$ are

$$
\lambda_{1,2}=\frac{1}{2}\left(\left(J_{11}+J_{22}\right) \pm \sqrt{\left(J_{11}-J_{22}\right)^{2}+4 J_{12} J_{21}}\right) \quad \text { and } \quad \lambda_{3}=J_{33}<0 .
$$

Since the product $J_{12} J_{21}<0$, we have

$$
\sqrt{\left(J_{11}-J_{22}\right)^{2}+4 J_{12} J_{21}}<\left|J_{11}-J_{22}\right| \leq\left|J_{11}\right|+\left|J_{22}\right| \leq\left|J_{11}+J_{22}\right|,
$$

so that $\operatorname{Re} \lambda_{1,2}<0$.

\section{ACKNOWLEDGEMENT}

Raimund Bürger is gratefully acknowledged for reading and commenting on the manuscript. SD acknowledges the Foundation for Engineering Scientific Research to the Memory of J. Gust. Richert, SWECO AB, Sweden. SF acknowledges the Crafoord Foundation, Sweden (grant 20110535). 


\section{REFERENCES}

[1] N. Alasino, M. C. Mussati, and N. Scenna. Wastewater treatment plant synthesis and design. Ind. Eng. Chem. Res., 46(23):7497-7512, 2007.

[2] S. Aouaouda, M. Chadli, M. Tarek Khadir, and T. Bouarar. Robust fault tolerant tracking controller design for unknown inputs T-S models with unmeasurable premise variables. $J$. Process Control, 2012. In press.

[3] A. Araujo, S. Gallani, M. Mulasy, and S. Skogestad. Sensitivity of optimal operation of an activated sludge process model. UKCC International Conference on Control, pages 259-264, Cardiff, UK, 3-5 September 2012.

[4] U. Attir and M. M. Denn. Dynamics and control of the activated sludge wastewater process. AIChE J., 24:693-698, 1978.

[5] N. G. Barton, C.-H. Li, and J. Spencer. Control of a surface of discontinuity in continuous thickeners. J. Austral. Math. Soc. Ser. B, 33:269-289, 1992.

[6] B. Boulkroune, M. Darouach, M. Zasadzinski, S. Gillé, and D. Fiorelli. A nonlinear observer design for an activated sludge wastewater treatment process. J. Process Control, 19(9):1558$1565,2009$.

[7] R. Bürger, S. Diehl, S. Farås, and I. Nopens. On reliable and unreliable numerical methods for the simulation of secondary settling tanks in wastewater treatment. Computers Chem. Eng., 41:93-105, 2012.

[8] R. Bürger, K. H. Karlsen, N. H. Risebro, and J. D. Towers. Numerical methods for the simulation of continuous sedimentation in ideal clarifier-thickener units. Int. J. Mineral Process., 73:209-228, 2004.

[9] R. Bürger, K. H. Karlsen, and J. D. Towers. A model of continuous sedimentation of flocculated suspensions in clarifier-thickener units. SIAM J. Appl. Math., 65:882-940, 2005.

[10] R. Bürger and A. Narváez. Steady-state, control, and capacity calculations for flocculated suspensions in clarifier-thickeners. Int. J. Mineral Process., 84(1-4):274-298, 2007.

[11] B. S. Cashion, T. M. Keinath, and W. W. Schuk. Control strategies for the activated sludge process. J. Water Pollution Control Federation, 51(4):815-833, 1979.

[12] J.-Ph. Chancelier, M. Cohen de Lara, C. Joannis, and F. Pacard. New insight in dynamic modelling of a secondary settler - II. Dynamical analysis. Water Res., 31(8):1857-1866, 1997.

[13] J.-Ph. Chancelier, M. Cohen de Lara, and F. Pacard. Analysis of a conservation PDE with discontinuous flux: A model of settler. SIAM J. Appl. Math., 54(4):954-995, 1994.

[14] S. Diehl. A conservation law with point source and discontinuous flux function modelling continuous sedimentation. SIAM J. Appl. Math., 56(2):388-419, 1996.

[15] S. Diehl. Dynamic and steady-state behaviour of continuous sedimentation. SIAM J. Appl. Math., 57(4):991-1018, 1997.

[16] S. Diehl. Operating charts for continuous sedimentation I: Control of steady states. J. Eng. Math., 41:117-144, 2001.

[17] S. Diehl. Operating charts for continuous sedimentation II: Step responses. J. Eng. Math., 53:139-185, 2005.

[18] S. Diehl. Operating charts for continuous sedimentation III: Control of step inputs. J. Eng. Math., 54:225-259, 2006.

[19] S. Diehl. Operating charts for continuous sedimentation IV: Limitations for control of dynamic behaviour. J. Eng. Math., 60:249-264, 2008.

[20] S. Diehl. A regulator for continuous sedimentation in ideal clarifier-thickener units. J. Eng. Math., 60:265-291, 2008.

[21] S. Diehl. The solids-flux theory - confirmation and extension by using partial differential equations. Water Res., 42(20):4976-4988, 2008.

[22] S. Diehl. A uniqueness condition for nonlinear convection-diffusion equations with discontinuous coefficients. J. Hyperbolic Differential Equations, 6:127-159, 2009.

[23] S. Diehl and S. Farås. A reduced-order ODE-PDE model for the activated sludge process in wastewater treatment: Classification and stability of steady states. Math. Models Meth. Appl. Sci. To appear.

[24] S. Diehl and S. Farås. Fundamental nonlinearities of the reactor-settler interaction in the activated sludge process. Water Sci. Tech., 66(1):28-35, 2012.

[25] D. Dochain. Design of adaptive controllers for non-linear stirred tank bioreactors: Extension to the MIMO situation. J. Process Control, 1(1):41-48, 1991. 
[26] D. Dochain. State and parameter estimation in chemical and biochemical processes: a tutorial. J. Process Control, 13(8):801-818, 2003.

[27] D. Dochain. Automatic Control of Bioprocesses. Control systems, robotics and manufacturing series. John Wiley \& Sons, 2010.

[28] G. A. Ekama. The role and control of sludge age in biological nutrient removal activated sludge systems. Water Sci. Tech., 61(7):1645-1652, 2010.

[29] X. Flores-Alsina, I. Rodriguez-Roda, G. Sin, and K.V. Gernaey. Multi-criteria evaluation of wastewater treatment plant control strategies under uncertainty. Water Res., 42(17):44854497, 2008.

[30] P. Georgieva and A. Ilchmann. Adaptive lambda-tracking control of activated sludge processes. Int. J. Control, 12:1247-1259, 2001.

[31] J. Guerrero, A. Guisasola, J. Comas, I. Rodríguez-Roda, and J. A. Baeza. Multi-criteria selection of optimum WWTP control setpoints based on microbiology-related failures, effluent quality and operating costs. Chem. Eng. J., 188(0):23-29, 2012.

[32] M. Henze, C. P. L. Grady, W. Gujer, G. v. R. Marais, and T. Matsuo. Activated sludge model no. 1. Technical Report 1, IAWQ, London, UK, 1987.

[33] A. Iratni, R. Katebi, and M. Mostefai. On-line robust nonlinear state estimators for nonlinear bioprocess systems. Commun. Nonlinear Sci. Numer. Simulat., 17(4):1739-1752, 2012.

[34] H. Ito. A dissipative approach to control of biological wastewater treatment plants based on entire nonlinear process models. In Proceedings of the 2004 American Control Conference, volume 6, pages 5489-5495, Boston, MA, 2004. IEEE.

[35] K. H. Karlsen, N. H. Risebro, and J. D. Towers. $L^{1}$ stability for entropy solutions of nonlinear degenerate parabolic convection-diffusion equations with discontinuous coefficients. Trans. Royal Norwegian Society Sci. Letters (Skr. K. Nor. Vidensk. Selsk.), 3:49, 2003.

[36] F. N. Koumboulis, N. D. Kouvakas, R. E. King, and A. Stathaki. Two-stage robust control of substrate concentration for an activated sludge process. ISA Transactions, 47(3):267-278, 2008.

[37] G. J. Kynch. A theory of sedimentation. Trans. Faraday Soc., 48:166-176, 1952.

[38] F. Lafont, E. Busvelle, and J.-P. Gauthier. An adaptive high-gain observer for wastewater treatment systems. J. Process Control, 21(6):893-900, 2011.

[39] T. T. Lee, F. Y. Wang, A. Islam, and R. B. Newell. Generic distributed parameter model control of a biological nutrient removal (BNR) activated sludge process. J. Process Control, 9(6):505-525, 1999.

[40] T. T. Lee, F. Y. Wang, and R. B. Newell. Robust model-order reduction of complex biological processes. J. Process Control, 12(2):807-821, 2002.

[41] T. T. Lee, F. Y. Wang, and R. B. Newell. Robust multivariable control of complex biological processes. J. Process Control, 14(2):193-209, 2004.

[42] T. T. Lee, F. Y. Wang, and R. B. Newell. Advances in distributed parameter approach to the dynamics and control of activated sludge processes for wastewater treatment. Water Res., 40(5):853-869, 2006.

[43] S. Marsili-Libelli. Dynamic modelling of sedimentation in the activated sludge process. Civil Eng. Syst., 10:207-224, 1993.

[44] G. Olsson. ICA and me - a subjective review. Water Res., 46(6):1585-1624, 2012.

[45] A. Rauh, M. Kletting, H. Aschemann, and E. P. Hofer. Robust controller design for bounded state and control variables and uncertain parameters using interval methods. International Conference on Control and Automation. ICCA '05., 2:777-782, 2005.

[46] P. Samuelsson, B. Halvarsson, and B. Carlsson. Interaction analysis and control structure selection in a wastewater treatment plant model. IEEE Trans. Control Systems Tech., 13(6):955-964, 2005

[47] G. Sin, K. V. Gernaey, M. B. Neumann, M. C. M. Van Loosdrecht, and W. Gujer. Global sensitivity analysis in wastewater treatment plant model applications: Prioritizing sources of uncertainty. Water Res., 45(2):639-651, 2011.

[48] H. Stehfest. An operational dynamic model of the final clarifier. Trans. Inst. Meas. Control, 6(3):160-164, 1984.

[49] A. Traoré, S. Grieu, F. Thiery, M Polit, and J. Colprim. Control of sludge height in a secondary settler using fuzzy algorithms. Comput. Chem. Eng., 30(8):1235-1243, 2006. 
[50] R. Tzoneva. Method for real time optimal control of the activated sludge process. In Mediterranean Conference on Control \& Automation, 200\%. (MED '07), pages 1-6, Athens, Greece, 2007.

[51] W. Zarrad, J. Harmand, M. Devisscher, and J. P. Steyer. Comparison of advanced control strategies for improving the monitoring of activated sludge processes. Control Eng. Practice, 12(3):323-333, 2004. 